\title{
Article \\ Genetic Diversity and Pathogenicity of Botryosphaeriaceae Species Associated with Symptomatic Citrus Plants in Europe
}

\author{
Jadson Diogo Pereira Bezerra ${ }^{1,2}$, , Pedro Wilhelm Crous ${ }^{2}$, Dalia Aiello ${ }^{3}$, , Maria Lodovica Gullino ${ }^{4}$, \\ Giancarlo Polizzi ${ }^{3}$ (D) and Vladimiro Guarnaccia $2,4,5, *$ (D)
}

1 Setor de Micologia, Departamento de Biociências e Tecnologia, Instituto de Patologia Tropical e Saúde Pública (IPTSP), Universidade Federal de Goiás (UFG), Rua 235, s/n, Setor Universitário, Goiânia 74605-050, Brazil; jadsondpb@gmail.com

2 Westerdijk Fungal Biodiversity Institute, Uppsalalaan 8, 3584 CT Utrecht, The Netherlands; p.crous@wi.knaw.nl

3 Dipartimento di Agricoltura, Alimentazione e Ambiente, sez. Patologia Vegetale, University of Catania, Via S. Sofia 100, 95123 Catania, Italy; dalia.aiello@unict.it (D.A.); gpolizzi@unict.it (G.P.)

4 Centre for Innovation in the Agro-Environmental Sector, AGROINNOVA, University of Torino, Largo Braccini 2, 10095 Grugliasco, Italy; marialodovica.gullino@unito.it

5 Department of Agricultural, Forest and Food Sciences (DISAFA), University of Torino, Largo Braccini 2, 10095 Grugliasco, Italy

* Correspondence: vladimiro.guarnaccia@unito.it

Citation: Bezerra, J.D.P.; Crous, P.W.; Aiello, D.; Gullino, M.L.; Polizzi, G.; Guarnaccia, V. Genetic Diversity and Pathogenicity of Botryosphaeriaceae Species Associated with Symptomatic Citrus Plants in Europe. Plants 2021, 10, 492. https://doi.org/10.3390/ plants10030492

Academic Editor: Takeshi Kanto

Received: 11 February 2021

Accepted: 2 March 2021

Published: 5 March 2021

Publisher's Note: MDPI stays neutral with regard to jurisdictional claims in published maps and institutional affiliations.

Copyright: (c) 2021 by the authors. Licensee MDPI, Basel, Switzerland. This article is an open access article distributed under the terms and conditions of the Creative Commons Attribution (CC BY) license (https:// creativecommons.org/licenses/by/ $4.0 /)$.

\begin{abstract}
This study represents the first survey studying the occurrence, genetic diversity, and pathogenicity of Botryosphaeriaceae species associated with symptomatic citrus species in citrusproduction areas in five European countries. Based on morphological features and phylogenetic analyses of internal transcribed spacer (ITS) of nuclear ribosomal DNA (nrDNA), translation elongation factor 1-alpha (TEF1) and $\beta$-tubulin (TUB2) genes, nine species were identified as belonging to the genera Diplodia, Dothiorella, Lasiodiplodia, and Neofusicoccum. Isolates of Neofusicoccum parvum and Diplodia pseudoseriata were the most frequently detected, while Dothiorella viticola had the widest distribution, occurring in four of the five countries sampled. Representative isolates of the nine Botryosphaeriaceae species used in the pathogenicity tests caused similar symptoms to those observed in nature. Isolates assayed were all re-isolated, thereby fulfilling Koch's postulates. Isolates of Diplodia pseudoseriata and Diplodia olivarum are recorded for the first time on citrus and all species found in our study, except N. parvum, are reported for the first time on citrus in Europe.
\end{abstract}

Keywords: Diplodia; Dothiorella; Lasiodiplodia; Neofusicoccum; pathogenic fungi; phylogeny

\section{Introduction}

Citrus production represents one of the most important fruit industries worldwide in terms of total yield. Greece, Italy, Portugal, and Spain are the most important European producers of citrus fruit [1]. In 2019, nearly 11 million tons of citrus was produced in Europe on approximately 515,000 ha [2]. Most canker diseases of citrus, as well as further fruit-tree crops, are caused by a broad range of fungal species that infect the wood mainly through winter pruning wounds and a subsequent colonization of vascular tissues [3]. Several abiotic and biotic factors are considered responsible for rots and gumming on the trunk and main branches in citrus. Frost damage, sunscald, or water distribution can promote the infection of numerous ascomycetes and basidiomycetes [4]. Several fungal infections involving twigs, branches and trunks of citrus caused by Colletotrichum and Diaporthe species were reported in different continents [5-9]. Guarnaccia and Crous [10] reported serious cankers developing in woody tissues of lemon trees caused by Diaporthe spp., often with a gummose exudate, causing serious blight and dieback. Canker diseases of citrus are also caused by other fungal genera such as Fusarium and Neocosmospora [11], Peroneutypa [12,13], and Phaeoacremonium [14]. Recently, significant attention has been 
dedicated to revising species and genera of Botryosphaeriaceae, which encompass species with a cosmopolitan distribution that are able to cause diseases of numerous plant species worldwide $[15,16]$.

Botryosphaeriaceae (Botryosphaeriales) include several species reported as endophytes, latent, and woody plant pathogens on a broad range of host [15-17]. This family has undergone significant revision after the adoption of molecular tools to resolve its taxonomy [15,16,18-23]. Recently, the taxonomy of Botryosphaeriaceae (and other families in Botryosphaeriales) has been reviewed by Phillips et al. [23] based on morphology of the sexual morphs, phylogenetic relationships on internal transcribed spacer (ITS) and $28 \mathrm{~S}$ large subunit (LSU) of nuclear ribosomal DNA (nrDNA) sequences and evolutionary divergence times. The authors highlighted the main findings made by Yang et al. [16] who included new families, genera, and species in Botryosphaeriales based on morphology and multi-marker phylogenetic analyses of a large collection of isolates. Currently, six families are accepted in Botryosphaeriales and 22 genera have been included in Botryosphaeriaceae [23-25].

The most common symptoms observed in association with species of Botryosphaeriaceae are twig, branch and trunk cankers, die-back, collar rot, root cankers, gummosis, decline and, in severe cases, plant death $[15,17]$. Plant infections mainly occur through natural openings or wounds, but these fungal species could also survive in latency. This ability could lead to their spread worldwide through asymptomatic plant material, seedlings and fruit, frequently circumventing the adopted quarantine measures [22]. Moreover, stress and non-optimal plant growth conditions consistently induce the expression of diseases associated with Botryosphaeriaceae species. Thus, global warming could increase plant stress and induce favourable conditions for the development of Botryosphaeriaceae diseases $[17,26,27]$. Species within the Botryosphaeriaceae represent a serious threat to different crops including major fruit, berry fruit and nut crops cultivated in sub-tropical, tropical, or temperate areas [22,28-30].

Several species of Diplodia (Di.), Dothiorella (Do.), Lasiodiplodia, Neofusicoccum, and Neoscytalidium (Ne.) have been previously reported to affect Citrus species [13,31-33]. For example, $\mathrm{Ne}$. dimidiatum has been reported causing citrus branch canker in California [13] and Italy [32]; Do. viticola, L. citricola, L. theobromae, and Ne. dimidiatum have been described in association with branch and trunk dieback of citrus trees in Iran [14,34] and Dothiorella spp. have been detected as causal agents of citrus gummosis in Tunisia [35]. Moreover, Di. seriata, Di. mutila, Do. viticola, L. mediterranea and L. mitidjana, have been recovered from symptomatic citrus trees in Algeria [33].

Considering the important economic value of Citrus spp., a large survey of Botryosphae riaceae affecting plants cultivated in the major citrus production areas of Europe was considered imperative. Identification in light of modern taxonomic concepts via morphological characterization and multi-marker DNA sequence data was necessary to adopt efficient control strategies against the pathogens that could affect these crops. Thus, several surveys have been conducted in Greece, Italy, Portugal, Spain, and Malta during 2015 and 2016. In particular, the aims of this study were to (1) conduct extensive surveys for sampling symptomatic plant materials; (2) obtain a broad collection of Botryosphaeriaceae isolates; (3) subject those isolates to DNA multi-marker sequence analyses combined with morphological characterization, and (4) evaluate the pathogenicity of the isolated species to citrus plants.

\section{Results}

\subsection{Field Sampling and Fungal Isolation}

In this study, the sampling focused on symptomatic plants of Citrus limon, C. reticulata, C. sinensis, C. sinensis $\times$ Poncirus trifoliata, and Microcitrus australasica. Samples were collected in 19 orchards (Table 1). Citrus trees showed various external disease symptoms, including partial or complete yellowing, wilting leaves and twigs, and dieback of branch tips, but also defoliation and branch decline. Canker and cracking of the bark associated 
with gummose exudate occurred on trunks and branches. Internal observation of infected branches revealed black to brown wood discoloration in cross-sections, wedge-shaped necrosis or irregular wood discoloration. Twigs were wilted and occasionally presenting sporocarps (Figure 1). Symptoms were detected in all the orchards and regions investigated. A total of 63 fungal isolates were collected and were found to be characterized by dark green to grey, fast-growing mycelium on MEA. Moreover, the isolates produced pycnidia on pine needles within 40 days, containing pigmented or hyaline conidia. According to these characteristics, the fungal isolates were classified as Botryosphaeriaceae spp. based on comparison with the previous generic descriptions [15]. Among the collected isolates, 18 were obtained from trunk cankers, 10 were associated with branch infections, and 35 from twig dieback (Table 2).

Table 1. Geographical sites investigated and sampled.

\begin{tabular}{ccc}
\hline Site & Locality & GPS Coordinates \\
\hline 1 & Algemesi (Spain) & $39^{\circ} 11^{\prime} 48.8^{\prime \prime} \mathrm{N}, 0^{\circ} 28^{\prime} 15.0^{\prime \prime} \mathrm{W}$ \\
2 & Alginet (Spain) & $39^{\circ} 15^{\prime} 36.3^{\prime \prime} \mathrm{N}, 0^{\circ} 27^{\prime} 28.9^{\prime \prime} \mathrm{W}$ \\
3 & Alhaurin El Grande (Spain) & $36^{\circ} 38^{\prime} 43.4^{\prime \prime} \mathrm{N}, 4^{\circ} 40^{\prime} 37.5^{\prime \prime} \mathrm{W}$ \\
4 & Alzira (Spain) & $39^{\circ} 09^{\prime} 25.1^{\prime \prime} \mathrm{N}, 0^{\circ} 29^{\prime} 26.6^{\prime \prime} \mathrm{W}$ \\
5 & Castellò (Spain) & $39^{\circ} 54^{\prime} 14.1^{\prime \prime} \mathrm{N}, 0^{\circ} 05^{\prime} 10.3^{\prime \prime} \mathrm{W}$ \\
6 & Estellencs (Spain) & $39^{\circ} 39^{\prime} 12.6^{\prime \prime} \mathrm{N}, 2^{\circ} 28^{\prime} 54.8^{\prime \prime} \mathrm{E}$ \\
7 & Faro (Portugal) & $37^{\circ} 03^{\prime} 45.5^{\prime \prime} \mathrm{N}, 7^{\circ} 55^{\prime} 02.8^{\prime \prime} \mathrm{W}$ \\
8 & Gozo (Malta) & $36^{\circ} 02^{\prime} 15.1^{\prime \prime} \mathrm{N}, 14^{\circ} 15^{\prime} 36.4^{\prime \prime} \mathrm{E}$ \\
9 & Gozo (Malta) & $36^{\circ} 03^{\prime} 18.5^{\prime \prime} \mathrm{N}, 14^{\circ} 15^{\prime} 35.7^{\prime \prime} \mathrm{E}$ \\
10 & Malaga (Spain) & $36^{\circ} 45^{\prime} 42.3^{\prime \prime} \mathrm{N}, 4^{\circ} 25^{\prime} 37.4^{\prime \prime} \mathrm{W}$ \\
11 & $37^{\circ} 46^{\prime} 05.7^{\prime \prime} \mathrm{N}, 15^{\circ} 11^{\prime} 40.7^{\prime \prime} \mathrm{E}$ \\
12 & Mascali (Italy) & $40^{\circ} 32^{\prime} 41.1^{\prime \prime} \mathrm{N}, 17^{\circ} 08^{\prime} 38.8^{\prime \prime} \mathrm{E}$ \\
13 & Massafra (Italy) & $38^{\circ} 25^{\prime} 49.0^{\prime \prime} \mathrm{N}, 21^{\circ} 16^{\prime} 49.9^{\prime \prime} \mathrm{E}$ \\
14 & Mastro (Greece) & $37^{\circ} 12^{\prime} 16.3^{\prime \prime} \mathrm{N}, 8^{\circ} 17^{\prime} 52.1^{\prime \prime} \mathrm{W}$ \\
15 & $39^{\circ} 35^{\prime} 18.8^{\prime \prime} \mathrm{N}, 0^{\circ} 23^{\prime} 40.5^{\prime \prime} \mathrm{W}$ \\
16 & $37^{\circ} 34^{\prime} 56.3^{\prime \prime} \mathrm{N}, 22^{\circ} 41^{\prime} 48.5^{\prime \prime} \mathrm{E}$ \\
17 & $40^{\circ} 06^{\prime} 30.2^{\prime \prime} \mathrm{N}, 16^{\circ} 37^{\prime} 04.6^{\prime \prime} \mathrm{E}$ \\
18 & Mesquita (Portugal) & $37^{\circ} 16^{\prime} 53.5^{\prime \prime} \mathrm{N}, 14^{\circ} 52^{\prime} 08.9^{\prime \prime} \mathrm{E}$ \\
19 & Moncada (Spain) & $37^{\circ} 09^{\prime} 50.7^{\prime \prime} \mathrm{N}, 8^{\circ} 23^{\prime} 21.7^{\prime \prime} \mathrm{W}$ \\
\hline
\end{tabular}




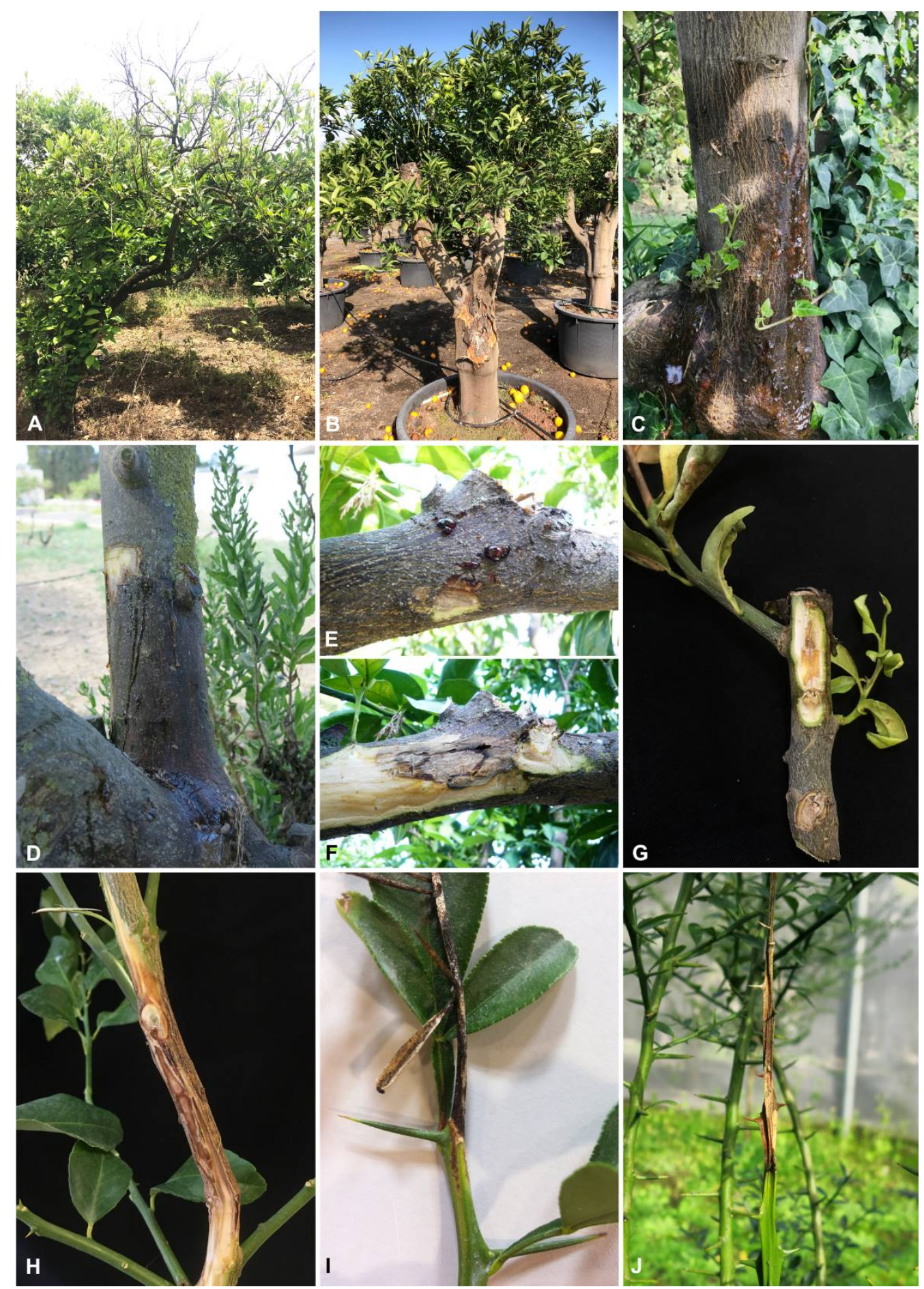

Figure 1. Symptoms on citrus tissues with associated Botryosphaeriacae species. (A) Branch decline in commercial lemon orchard. (B) Trunk canker and bark cracking of C. sinensis. (C,D) Trunk and branch canker with gummosis of $C$. sinensis plants. (E,F) External cracking with gummosis and internal wood discoloration of the same affected branch of $C$. reticulata plant. $(\mathbf{G}, \mathbf{H})$ Internal wood discoloration and branch blight of C. limon. (I) Twig dieback of young C. sinensis $\times$ P. trifoliata and $M$. australasica (J) plants. 


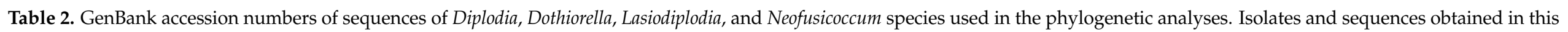
study are given in bold.

\begin{tabular}{|c|c|c|c|c|c|c|}
\hline \multirow{2}{*}{ Species } & \multirow{2}{*}{ Strains ${ }^{1}$} & \multirow{2}{*}{ Host/Substrate } & \multirow{2}{*}{ Country } & \multicolumn{3}{|c|}{ GenBank Numbers $^{2}$} \\
\hline & & & & ITS & TEF1 & TUB2 \\
\hline Botryosphaeria dothidea & CBS 115476 = CMW 8000, ex-epitype & Prunus sp. & Switzerland & AY236949 & AY236898 & AY236927 \\
\hline Diplodia africana & CBS 120835 = CPC 5908, ex-type & Prunus persica, stem canker & South Africa & EF445343 & EF445382 & KF766129 \\
\hline Di. agrifolia & CBS 132777 = UCR732, ex-type & Quercus agrifolia, cankered branch & USA: California & JN693507 & JQ517317 & JQ411459 \\
\hline Di. allocellula & CBS $130408=$ CMW 36468, ex-type & Acacia karroo, healthy branches & South Africa & JQ239397 & JQ239384 & JQ239378 \\
\hline Di. bulgarica & CBS 124254 = CAP332, ex-type & Malus sylvestris & Bulgaria & GQ923853 & GQ923821 & - \\
\hline Di. citricarpa & $\begin{array}{c}\text { CBS } 124715=\text { CJA } 131=\text { IRAN 1578C, } \\
\text { ex-type }\end{array}$ & Citrus sp., twigs & Iran & KF890207 & KF890189 & KX464784 \\
\hline Di. corticola & CBS 112549 = CAP 134, ex-type & Quercus suber & Portugal & AY259100 & AY573227 & DQ458853 \\
\hline Di. crataegicola & MFLU 15-13112, ex-type & Crataegus sp. & Italy & KT290244 & KT290248 & KT290246 \\
\hline Di. cupressi & CBS 168.87, ex-type & Cupressus sempervirens, canker & Israel & DQ458893 & DQ458878 & DQ458861 \\
\hline Di. eriobotryicola & CBS 140851 = BN-21, ex-type & Eriobotrya japonica & Spain & KT240355 & KT240193 & MG015806 \\
\hline Di. estuarina & CMW 41231 & Avicennia marina & South Africa & KP860831 & KP860676 & KP860754 \\
\hline Di. gallae & CBS 211.25 & Quercus sp., fruit & - & KX464090 & KX464564 & KX464795 \\
\hline Di. gallae & CBS 212.25 & Quercus sp., gall & - & KX464091 & KX464565 & KX464796 \\
\hline Di. gallae & CBS 213.25 & Quercus sp., gall & - & KX464092 & KX464566 & KX464797 \\
\hline Di. malorum & CBS $124130=$ CAP271, ex-type & Malus sylvestris & Portugal & GQ923865 & GQ923833 & - \\
\hline Di. mutila & CPC 26977 & Citrus sinensis, twig & Greece & MW413831 & MW419149 & MW419212 \\
\hline Di. mutila & CBS 112553 = CAP 062 & Vitis vinifera & Portugal & AY259093 & AY573219 & DQ458850 \\
\hline Di. mutila & $\begin{array}{c}\text { CBS } 121862=\text { PD } 03708098, \text { ex-type of Di. } \\
\text { pyri }\end{array}$ & Pyrus sp. & The Netherlands & KX464093 & KX464567 & KX464799 \\
\hline Di. neojuniperi & CPC 22753 = B0031, ex-type & Juniperus chinensis & Thailand & KM006431 & KM006462 & - \\
\hline Di. olivarum & CPC 27855 & Citrus sinensis,branch & Malta & MW413832 & MW419150 & MW419213 \\
\hline Di. olivarum & CPC 27856 & Citrus sinensis,branch & Malta & MW413833 & MW419151 & MW419214 \\
\hline
\end{tabular}


Table 2. Cont.

\begin{tabular}{|c|c|c|c|c|c|c|}
\hline \multirow{2}{*}{ Species } & \multirow{2}{*}{ Strains ${ }^{1}$} & \multirow{2}{*}{ Host/Substrate } & \multirow{2}{*}{ Country } & \multicolumn{3}{|c|}{ GenBank Numbers $^{2}$} \\
\hline & & & & ITS & TEF1 & TUB2 \\
\hline Di. olivarum & CBS 121886 & Olea europaea & Italy & EU392301 & EU392278 & - \\
\hline Di. pseudoseriata & CBS 124906, ex-type & Blepharocalyx salicifolius & Uruguay & EU080927 & EU863181 & MG015820 \\
\hline Di. pseudoseriata & СРС 27963 & Citrus sinensis, twig & Portugal & MW413834 & MW419152 & MW419215 \\
\hline Di. pseudoseriata & СРС 27964 & Citrus sinensis, twig & Portugal & MW413835 & MW419153 & MW419216 \\
\hline Di.pseudoseriata & CPC 27965 & Citrus sinensis, twig & Portugal & MW413836 & MW419154 & MW419217 \\
\hline Di.pseudoseriata & CPC 27966 & Citrus sinensis, twig & Portugal & MW413837 & MW419155 & MW419218 \\
\hline Di. pseudoseriata & CРC 27967 & Citrus sinensis, twig & Portugal & MW413838 & MW419156 & MW419219 \\
\hline Di.pseudoseriata & CPC 28084 & Citrus reticulata, twig & Spain & MW413839 & MW419157 & MW419220 \\
\hline Di. pseudoseriata & СРC 28086 & Citrus reticulata, twig & Spain & MW413840 & MW419158 & MW419221 \\
\hline Di.pseudoseriata & CPC 28087 & Citrus reticulata, twig & Spain & MW413841 & MW419159 & MW419222 \\
\hline Di.pseudoseriata & CPC 28092 & Citrus limon, twig & Spain & MW413842 & MW419160 & MW419223 \\
\hline Di.pseudoseriata & CPC 28094 & Citrus limon, twig & Spain & MW413844 & MW419162 & MW419225 \\
\hline Di. pseudoseriata & СРС 28095 & Citrus limon, twig & Spain & MW413845 & MW419163 & MW419226 \\
\hline Di.pseudoseriata & CPC 28099 & Citrus reticulata, twig & Spain & MW413846 & MW419164 & MW419227 \\
\hline Di. pseudoseriata & CРC 28100 & Citrus reticulata, twig & Spain & MW413847 & MW419165 & MW419228 \\
\hline Di. pseudoseriata & CPC 28102 & Citrus reticulata, twig & Spain & MW413848 & MW419166 & MW419229 \\
\hline Di. pseudoseriata & BL132 & Fraxinus angustifolia & Italy & KF307720 & KF318767 & MG015810 \\
\hline Di. pseudoseriata & CBS 140350 , ex-type of Di. insularis & Pistacia lentiscus & Italy & KX833072 & KX833073 & MG015809 \\
\hline Di. pseudoseriata & $\begin{array}{c}\text { CBS } 124931=\text { CMW22627, ex-type of Di. } \\
\text { alatafructa }\end{array}$ & Pterocarpus angolensis, bark wound & South Africa & FJ888460 & FJ888444 & MG015799 \\
\hline Di. quercivora & CBS 133852 = BL8, ex-type & Quercus canariensis & Tunisia & JX894205 & JX894229 & MG015821 \\
\hline
\end{tabular}


Table 2. Cont.

\begin{tabular}{|c|c|c|c|c|c|c|}
\hline \multirow{2}{*}{ Species } & \multirow{2}{*}{ Strains 1} & \multirow{2}{*}{ Host/Substrate } & \multirow{2}{*}{ Country } & \multicolumn{3}{|c|}{ GenBank Numbers $^{2}$} \\
\hline & & & & ITS & TEF1 & TUB2 \\
\hline Di. rosulata & CBS 116470, ex-type & Prunus africana & Ethiopia & EU430265 & EU430267 & EU673132 \\
\hline Di. sapinea & CBS 393.84, ex-epitype & Pinus nigra, cones & Netherlands & DQ458895 & DQ458880 & DQ458863 \\
\hline Di. sapinea & $\begin{aligned} \text { CBS } 124462= & \text { CAP273, ex-type of Di. } \\
& \text { intermedia }\end{aligned}$ & Malus sylvestris & Portugal & GQ923858 & GQ923826 & - \\
\hline Di. sapinea & $\begin{array}{c}\text { CBS } 141915=\text { NB7, ex-type of Di. } \\
\text { rosacearum }\end{array}$ & Eriobotrya japonica & Italy & KT956270 & KU378605 & MG015823 \\
\hline Di. scrobiculata & $\begin{aligned} \text { CBS } 118110= & \text { CMW } 189=\text { BOT 1195, } \\
& \text { ex-type }\end{aligned}$ & Pinus banksiana & USA: Wisconsin & AY253292 & AY624253 & AY624258 \\
\hline Di. seriata & $\begin{array}{c}\text { CBS } 112555=\text { HAP } 052=\text { CAP 063, } \\
\text { ex-epitype }\end{array}$ & Vitis vinifera, dead stems & Portugal & AY259094 & AY573220 & DQ458856 \\
\hline Di. seriata & СРC 28088 & Citrus reticulata,twig & Spain & MW413849 & MW419167 & MW419230 \\
\hline Di. seriata & СРC 28089 & Citrus reticulata,twig & Spain & MW413850 & MW419168 & MW419231 \\
\hline Di. seriata & CPC 28090 & Citrus reticulata,twig & Spain & MW413851 & MW419169 & MW419232 \\
\hline Di. seriata & СРC 28096 & Citrus sinensis,twig & Spain & MW413853 & MW419171 & MW419234 \\
\hline Di. seriata & СРC 28097 & Citrus sinensis,twig & Spain & MW413854 & MW419172 & MW419235 \\
\hline Di. seriata & CPC 28098 & Citrus sinensis,twig & Spain & MW413855 & MW419173 & MW419236 \\
\hline Di. seriata & CPC 28101 & Citrus reticulata,twig & Spain & MW413856 & MW419174 & MW419237 \\
\hline Di. seriata & СРC 28103 & Citrus reticulata,twig & Spain & MW413857 & MW419175 & MW419238 \\
\hline Di. seriata & CBS 119049 & Vitis sp. & Italy & DQ458889 & DQ458874 & DQ458857 \\
\hline Di. subglobosa & CBS 124133 = JL453, ex-type & Lonicera nigra & Spain & GQ923856 & GQ923824 & - \\
\hline Di. tsugae & CBS 418.64 = IMI 197143, ex-isotype & Tsuga heterophylla & Canada & DQ458888 & DQ458873 & DQ458855 \\
\hline
\end{tabular}


Table 2. Cont.

\begin{tabular}{|c|c|c|c|c|c|c|}
\hline \multirow{2}{*}{ Species } & \multirow{2}{*}{ Strains ${ }^{1}$} & \multirow{2}{*}{ Host/Substrate } & \multirow{2}{*}{ Country } & \multicolumn{3}{|c|}{ GenBank Numbers $^{2}$} \\
\hline & & & & ITS & TEF1 & TUB2 \\
\hline Dothiorella alpina & CGMCC 3-18001, ex-type & Platycladus orientalis & China & KX499645 & KX499651 & - \\
\hline Do. brevicollis & CBS 130411 = CMW 36463, ex-type & Acacia karroo, healthy branches & South Africa & JQ239403 & JQ239390 & JQ239371 \\
\hline Do. capri-amissi & $\begin{aligned} \text { CBS } 121763= & \text { CMW } 25403=\text { CAMS 1158, } \\
& \text { ex-paratype }\end{aligned}$ & Acacia erioloba & South Africa & EU101323 & EU101368 & KX464850 \\
\hline Do. capri-amissi & $\begin{array}{c}\text { CBS } 121878=\text { CMW } 25404=\text { CAMS 1159, } \\
\text { ex-type }\end{array}$ & Acacia erioloba & South Africa & EU101324 & EU101369 & KX464851 \\
\hline Do. casuarinae & CBS 120688 = CMW 4855, ex-type & Casuarina sp. & $\begin{array}{l}\text { Australia: Australian } \\
\text { Capital Territory }\end{array}$ & DQ846773 & DQ875331 & DQ875340 \\
\hline Do. casuarinae & CBS 120689 = CMW 4856, ex-paratype & Casuarina sp. & $\begin{array}{l}\text { Australia: Australian } \\
\text { Capital Territory }\end{array}$ & DQ846772 & DQ875332 & DQ875339 \\
\hline Do. citricola & CBS $124728=$ ICMP 16827 & Citrus sinensis & New Zealand & EU673322 & EU673289 & KX464852 \\
\hline Do. citricola & CBS $124729=$ ICMP 16828, ex-type & Citrus sinensis, twigs & New Zealand & EU673323 & EU673290 & KX464853 \\
\hline Do. dulcispinae & $\begin{array}{c}\text { CBS } 121764=\text { CMW } 25406=\text { CAMS 1159, } \\
\text { ex-paratype of Dothiorella oblonga }\end{array}$ & Acacia mellifera & Namibia & EU101299 & EU101344 & KX464854 \\
\hline Do. dulcispinae & CBS 130413 = CMW 36460, ex-type & Acacia karroo, dieback branches & South Africa & JQ239400 & JQ239387 & JQ239373 \\
\hline Do. iberica & CBS $113188=$ DA-1 & Quercus suber, branch canker & Spain & AY573198 & EU673278 & EU673097 \\
\hline Do. iberica & CBS $113189=$ DE-14 & Quercus ilex, branch canker & Spain & AY573199 & AY573230 & KX464855 \\
\hline Do. iberica & CBS 115041 = CAP 145, ex-type & Quercus ilex, dead twigs & Spain & AY573202 & AY573222 & EU673096 \\
\hline Do. iranica & CBS $124722=$ CJA $153=$ IRAN 1587C & Olea sp., twigs & Iran & KC898231 & KC898214 & KX464856 \\
\hline Do. longicollis & CBS 122066 = CMW 26164 & Terminalia sp. & $\begin{array}{l}\text { Australia: Western } \\
\text { Australia }\end{array}$ & EU144052 & EU144067 & KX464857 \\
\hline
\end{tabular}


Table 2. Cont.

\begin{tabular}{|c|c|c|c|c|c|c|}
\hline \multirow{2}{*}{ Species } & \multirow{2}{*}{ Strains ${ }^{1}$} & \multirow{2}{*}{ Host/Substrate } & \multirow{2}{*}{ Country } & \multicolumn{3}{|c|}{ GenBank Numbers $^{2}$} \\
\hline & & & & ITS & TEF1 & TUВ2 \\
\hline Do. longicollis & CBS 122067 = CMW 26165 & Lysiphyllum cunninghamii & $\begin{array}{l}\text { Australia: Western } \\
\text { Australia }\end{array}$ & EU144053 & EU144068 & KX464858 \\
\hline Do. longicollis & CBS $122068=$ CMW 26166, ex-type & Lysiphyllum cunninghamii & $\begin{array}{l}\text { Australia: Western } \\
\text { Australia }\end{array}$ & EU144054 & EU144069 & KF766130 \\
\hline Do. mangifericola & $\begin{array}{c}\text { CBS } 124727=\text { IRAN } 1584 C=\text { CJA 261, } \\
\text { ex-type }\end{array}$ & Mangifera indica, twigs & Iran & KC898221 & KX464614 & - \\
\hline Do. moneti & WAC 13154 = MUCC 505, ex-type & Acacia rostellifera, healthy stem & $\begin{array}{l}\text { Australia: Western } \\
\text { Australia }\end{array}$ & EF591920 & EF591971 & EF591954 \\
\hline Do. neclivorem & DAR 80992, ex-type & Vitis vinifera $\mathrm{cv}$. Chardonnay, berries & $\begin{array}{l}\text { Australia: New South } \\
\text { Wales }\end{array}$ & KJ573643 & KJ573640 & KJ577551 \\
\hline Do. oblonga & $\begin{array}{c}\text { CBS } 121765=\text { CMW } 25407=\text { CAMS 1162, } \\
\text { ex-type }\end{array}$ & Acacia mellifera & South Africa & EU101300 & EU101345 & KX464862 \\
\hline Do. oblonga & $\begin{aligned} \text { CBS } 121766= & \text { CMW 25408 = CAMS 1163, } \\
& \text { ex-paratype }\end{aligned}$ & Acacia mellifera & South Africa & EU101301 & EU101346 & KX464863 \\
\hline Do. omnivora & CBS 392.80 & - & France & KX464133 & KX464626 & KX464897 \\
\hline Do. omnivora & CBS $124716=$ CJA $241=$ IRAN 1573C & Juglans regia, twigs & Iran & KC898232 & KC898215 & KX464864 \\
\hline Do. omnivora & CBS 242.51 & - & Italy & EU673317 & EU673284 & EU673105 \\
\hline Do. omnivora & CBS 188.87 & Juglans regia & France & EU673316 & EU673283 & EU673119 \\
\hline Do. parva & $\begin{aligned} \text { CBS } 124720= & \text { CJA } 27=\text { IRAN } 1579 C, \\
& \text { ex-type }\end{aligned}$ & Corylus sp., twigs & Iran & КC898234 & KC898217 & KX464866 \\
\hline Do. parva & CBS $124721=$ CJA 35 & Corylus sp., twigs & Iran & KX464123 & KX464615 & KX464867 \\
\hline Do. parva & CBS 125580 & Corylus avellana, branches & Austria & KX464124 & KX464616 & KX464868 \\
\hline Do. plurivora & $\begin{array}{c}\text { CBS } 124724=\text { CJA } 254=\text { IRAN } 1557 \text { C, } \\
\text { ex-type }\end{array}$ & Citrus sp., twigs & Iran & KC898225 & KC898208 & KX464874 \\
\hline Do. pretoriensis & CBS 130404 = CMW 36480, ex-type & Acacia karroo, branches with dieback & South Africa & JQ239405 & JQ239392 & JQ239376 \\
\hline
\end{tabular}


Table 2. Cont.

\begin{tabular}{|c|c|c|c|c|c|c|}
\hline \multirow{2}{*}{ Species } & \multirow{2}{*}{ Strains ${ }^{1}$} & \multirow{2}{*}{ Host/Substrate } & \multirow{2}{*}{ Country } & \multicolumn{3}{|c|}{ GenBank Numbers $^{2}$} \\
\hline & & & & ITS & TEF1 & TUB2 \\
\hline Do. prunicola & $\begin{array}{c}\text { CBS } 124723=\text { CAP } 187=\text { IRAN } 1541 C, \\
\text { ex-type }\end{array}$ & Prunus dulcis, necrotic twigs & Portugal & EU673313 & EU673280 & EU673100 \\
\hline Do. rosulata & $\begin{array}{c}\text { CBS } 121760=\text { CMW } 25389=\text { CAMS 1444, } \\
\text { ex-type }\end{array}$ & Acacia karroo & Namibia & KF766227 & EU101335 & KX464877 \\
\hline Do. rosulata & $\begin{aligned} \text { CBS } 121761= & \text { CMW } 25392=\text { CAMS 1147, } \\
& \text { ex-paratype }\end{aligned}$ & Acacia mellifera & South Africa & EU101293 & EU101338 & KX464878 \\
\hline Do. rosulata & CBS 121762 = CMW 25395 = CAMS 1150 & Acacia mellifera & South Africa & EU101319 & EU101364 & KX464879 \\
\hline Do. rosulata & CBS 500.72 & Medicago sativa, stubble & South Africa & EU673318 & EU673285 & EU673118 \\
\hline Do. santali & WAC 13155 = MUCC 509, ex-type & Santalum acuminatum, healthy stem & $\begin{array}{l}\text { Australia: Western } \\
\text { Australia }\end{array}$ & EF591924 & EF591975 & EF591958 \\
\hline Do. sarmentorum & IMI $63581 \mathrm{~b}$, ex-type of Bot. sarmentorum & Ulmus sp. & UK: England & AY573212 & AY573235 & EU673102 \\
\hline Do. sempervirentis & IRAN 1581C = CBS 124719 & Cupressus sempervirens & Iran & KC898237 & KC898220 & KX464885 \\
\hline Do. sempervirentis & $\begin{aligned} \text { IRAN } 1583 C= & \text { CBS } 124718=\text { CJA 264, } \\
& \text { ex-type }\end{aligned}$ & Cupressus sempervirens, twigs & Iran & KC898236 & KC898219 & KX464884 \\
\hline Do. striata & CBS 124730 = ICMP 16819 & Citrus sinensis, twigs & New Zealand & EU673320 & EU673287 & EU673142 \\
\hline Do. striata & CBS 124731 = ICMP 16824, ex-type & Citrus sinensis, twigs & New Zealand & EU673321 & EU673288 & EU673143 \\
\hline Do. thailandica & $\begin{array}{c}\text { CBS } 133991=\text { CPC } 21557=\text { MFLUCC } \\
\text { 11-0438, ex-type of Auerswaldia dothiorella }\end{array}$ & Dead bamboo culm & Thailand & JX646796 & JX646861 & JX646844 \\
\hline Do. thripsita & CBS 125445 = BRIP 51876a, ex-type & $\begin{array}{c}\text { Acacia harpophylla, dead branches, } \\
\text { petioles \& leaves }\end{array}$ & Australia: Queensland & KJ573642 & KJ573639 & KJ577550 \\
\hline Do. uruguayensis & $\begin{aligned} \text { CBS } 124908= & \text { CMW } 26763=\text { UY672, } \\
& \text { ex-type }\end{aligned}$ & Hexachlamis edulis & Uruguay & EU080923 & EU863180 & KX464886 \\
\hline Do. vidmadera & CBS 621.74 & Pyrus communis, leaf & Switzerland & KX464129 & KX464621 & KX464887 \\
\hline Do. vidmadera & CBS 725.79 & Pyrus malus, dead wood and buds & Switzerland & KX464130 & KX464622 & KX464888 \\
\hline Do. vinea-gemmae & DAR 81012, ex-type & $\begin{array}{l}\text { Vitis vinifera } \mathrm{cv} \text {. Chardonnay, dormant } \\
\text { buds }\end{array}$ & $\begin{array}{l}\text { Australia: New South } \\
\text { Wales }\end{array}$ & KJ573644 & KJ573641 & KJ577552 \\
\hline
\end{tabular}


Table 2. Cont.

\begin{tabular}{|c|c|c|c|c|c|c|}
\hline \multirow{2}{*}{ Species } & \multirow{2}{*}{ Strains ${ }^{1}$} & \multirow{2}{*}{ Host/Substrate } & \multirow{2}{*}{ Country } & \multicolumn{3}{|c|}{ GenBank Numbers $^{2}$} \\
\hline & & & & ITS & TEF1 & TUB2 \\
\hline Do. viticola & CBS 117009, ex-type & $\begin{array}{l}\text { Vitis vinifera cv. Garnatxa negra, } \\
\text { pruned canes }\end{array}$ & Spain & AY905554 & AY905559 & EU673104 \\
\hline Do. viticola & DAR 80529 , ex-type of $D$. westralis & $\begin{array}{l}\text { Vitis vinifera cv. Cabernet Sauvignon, } \\
\text { discarded canes }\end{array}$ & $\begin{array}{l}\text { Australia: Western } \\
\text { Australia }\end{array}$ & HМ009376 & HM800511 & HM800519 \\
\hline Do. viticola & CPC 26174 & Citrus sinensis, twig & Italy & MW413858 & MW419176 & MW419239 \\
\hline Do. viticola & CPC 27081 & Citrus sinensis, twig & Italy & MW413860 & MW419178 & MW419241 \\
\hline Do. viticola & CPC 27106 & Citrus aurantium, twig & Spain & MW413861 & MW419179 & MW419242 \\
\hline Do. viticola & CPC 27123 & Citrus sinensis, branch & Italy & MW413862 & MW419180 & MW419243 \\
\hline Do. viticola & CPC 27125 & Citrus sinensis, branch & Italy & MW413863 & MW419181 & MW419244 \\
\hline Do. viticola & CРC 27703 & Citrus sinensis, branch & Spain & MW413864 & MW419182 & MW419245 \\
\hline Do. viticola & CPC 27707 & Citrus sinensis, branch & Greece & MW413865 & MW419183 & MW419246 \\
\hline Do. yunnana & CGMCC 3-17999, ex-type & Camellia sp. & China & KX499643 & KX499649 & - \\
\hline Do. yunnana & CGMCC 3-18000 & Camellia sp. & China & KX499644 & KX499650 & - \\
\hline Dothiorella sp. & CBS 121783 = CMW 25432 = CAMS 1187 & Acacia mearnsii & South Africa & EU101333 & EU101378 & KX464859 \\
\hline Dothiorella sp. & CBS 121784 = CMW 25430 = CAMS 1185 & Acacia mearnsii & South Africa & EU101331 & EU101376 & KX464860 \\
\hline Dothiorella sp. & CBS 121785 = CMW 25433 = CAMS 1188 & Acacia mearnsii & South Africa & EU101334 & EU101379 & KX464861 \\
\hline 'Lasiodiplodia americana' & CERC 1961 = CFCC 50065, ex-type & Pistacia vera cv. Kerman, twigs & USA: Arizona & KP217059 & KP217067 & KP217075 \\
\hline L. avicenniae & CMW 41467 = CBS 139670, ex-type & Avicennia marina & South Africa & KP860835 & KP860680 & KP860758 \\
\hline L. brasiliense & CMM 4015 = URM 7118, ex-type & Mangifera indica, stems & Brazil & JX464063 & JX464049 & - \\
\hline L. bruguierae & CMW 41470 = CBS 139669, ex-type & Bruguiera gymnorrhiza & South Africa & NR_147358 & KP860678 & KP860756 \\
\hline L. citricola & $\begin{aligned} \text { CBS } 124707= & \text { IRAN } 1522 C=\text { CJA } 72, \\
& \text { ex-type }\end{aligned}$ & Citrus sp., twigs & Iran & GU945354 & GU945340 & KP872405 \\
\hline
\end{tabular}


Table 2. Cont.

\begin{tabular}{|c|c|c|c|c|c|c|}
\hline \multirow{2}{*}{ Species } & \multirow{2}{*}{ Strains ${ }^{1}$} & \multirow{2}{*}{ Host/Substrate } & \multirow{2}{*}{ Country } & \multicolumn{3}{|c|}{ GenBank Numbers $^{2}$} \\
\hline & & & & ITS & TEF1 & TUB2 \\
\hline L. crassispora & $\begin{array}{c}\text { CBS } 118741=\text { WAC } 12533=\text { CMW 14691, } \\
\text { ex-type }\end{array}$ & Santalum album & $\begin{array}{l}\text { Australia: Western } \\
\text { Australia }\end{array}$ & DQ103550 & EU673303 & EU673133 \\
\hline L. crassispora & $\begin{array}{c}\text { CBS } 121770=\text { CMW } 25414=\text { CAMS } 1169, \\
\text { ex-type of L. pyriformis }\end{array}$ & Acacia mellifera & Namibia & EU101307 & EU101352 & - \\
\hline L. endophytica & $\begin{array}{c}\text { MFLUCC 18-1121 = KUMCC 17-0233, } \\
\text { ex-type }\end{array}$ & Magnolia candolii, fresh leaves & China & MK501838 & MK584572 & MK550606 \\
\hline L. egyptiacae & CBS 130992 = BOT-10, ex-type & Mangifera indica, leaf & Egypt & JN814397 & JN814424 & - \\
\hline L. euphorbicola & CMM 3609, ex-type of L. euphorbicola & Jatropha curcas, collar and root rot & Brazil & KF234543 & KF226689 & KF254926 \\
\hline L. gilanensis & CBS 124704 = IRAN 1523C, ex-type & Citrus sp., fallen twigs & Iran & GU945351 & GU945342 & KP872411 \\
\hline L. gilanensis & $\begin{aligned} \text { CBS } 128311= & \text { UCD } 2193 \mathrm{MO}, \text { ex-type of } L . \\
& \text { missouriana }\end{aligned}$ & $\begin{array}{c}\text { Wedge-shape canker of grapevine cv. } \\
\text { Catawba (complex hybrid of North } \\
\text { America Vitis species and Vitis } \\
\text { vinifera) }\end{array}$ & USA: Missouri & HQ288225 & HQ288267 & - \\
\hline L. gonubiensis & CBS 115812 = CMW 14077, ex-type & Syzygium cordatum, twigs and leaves & South Africa & AY639595 & DQ103566 & DQ458860 \\
\hline L. gravistriata & CMM 4564, ex-type & Anacardium humile & Brazil & KT250949 & KT250950 & - \\
\hline L. hormozganensis & CBS $124709=$ IRAN 1500C, ex-type & Olea sp., twigs & Iran & GU945355 & GU945343 & KP872413 \\
\hline L. iraniensis & CBS $124710=$ IRAN 1520C, ex-type & Salvadora persica, twigs & Iran & GU945346 & GU945334 & KP872415 \\
\hline L. iraniensis & CMM 3610, ex-type of L. jatrophicola & Jatropha curcas, collar and root rot & Brazil & KF234544 & KF226690 & KF254927 \\
\hline L. laeliocattleyae & $\begin{array}{l}\text { CBS 167.28, ex-type of Diplodia } \\
\text { laeliocattleyae }\end{array}$ & Laeliocattleya & Italy & KU507487 & KU507454 & - \\
\hline L. lignicola & MFLUCC 11-0435 = CBS 134112, ex-type & On dead wood & Thailand & JX646797 & KU887003 & JX646845 \\
\hline L. lignicola & CBS 342.78, ex-type of L. sterculiae & Sterculia oblonga & Germany & KX464140 & KX464634 & KX464908 \\
\hline L. macrospora & CMM 3833, ex-type & Jatropha curcas, collar and root rot & Brazil & KF234557 & KF226718 & KF254941 \\
\hline 'L. magnoliae' & $\begin{array}{c}\text { MFLUCC 18-0948 = KUMCC 17-0198, } \\
\text { ex-type }\end{array}$ & Magnolia candolii, dead leaves & China & MK499387 & MK568537 & MK521587 \\
\hline L. mahajangana & CBS 124927 = CMW27801, ex-type & Terminalia catappa, healthy branches & Madagascar & FJ900595 & FJ900641 & FJ900630 \\
\hline
\end{tabular}


Table 2. Cont.

\begin{tabular}{|c|c|c|c|c|c|c|}
\hline \multirow{2}{*}{ Species } & \multirow{2}{*}{ Strains $^{1}$} & \multirow{2}{*}{ Host/Substrate } & \multirow{2}{*}{ Country } & \multicolumn{3}{|c|}{ GenBank Numbers $^{2}$} \\
\hline & & & & ITS & TEF1 & TUB2 \\
\hline L. mahajangana & CMM 1325, ex-type of L. caatinguensis & Citrus sinensis & Brazil & KT154760 & KT008006 & KT154767 \\
\hline L. mahajangana & CBS $137785=$ BL104, ex-type of L. exigua & Retama raetam, branch canker & Tunisia & KJ638317 & KJ638336 & - \\
\hline L. margaritacea & $\begin{array}{c}\text { CBS } 122519=\text { CMW } 26162=\text { MOZ 11A } \\
\text { ex-type }\end{array}$ & Adansonia gibbosa & $\begin{array}{l}\text { Australia: Western } \\
\text { Australia }\end{array}$ & EU144050 & EU144065 & KX464903 \\
\hline L. mediterranea & CBS 137783 = BL1, ex-type & Quercus ilex, branch canker & Italy & KJ638312 & KJ638331 & - \\
\hline L. parva & CBS 456.78, ex-type & Cassava-field soil & Colombia & EF622083 & EF622063 & KP872419 \\
\hline L. plurivora & CBS 120832 = CPC 5803, ex-type & Prunus salicina, wood canker & South Africa & EF445362 & EF445395 & KP872421 \\
\hline L. pontae & CMM 1277, ex-type & Spondias purpurea & Brazil & KT151794 & KT151791 & KT151797 \\
\hline L.pseudotheobromae & CBS 116459, ex-type & Gmelina arborea & Costa Rica & EF622077 & EF622057 & EU673111 \\
\hline L. rubropurpurea & $\begin{array}{c}\text { CBS } 118740=\text { WAC } 12535=\text { CMW 14700, } \\
\text { ex-type }\end{array}$ & Eucalyptus grandis, canker & Australia & DQ103553 & EU673304 & EU673136 \\
\hline L. thailandica & CBS $138760=$ CPC 22795, ex-type & Mangifera indica, twigs & Thailand & KJ193637 & KJ193681 & - \\
\hline L. theobromae & CBS $111530=$ CPC 2095 = JT 695 & Leucospermum sp. & USA: Hawaii & EF622074 & EF622054 & - \\
\hline L. theobromae & CPC 27881 & Citrus sinensis, trunk & Malta & MW413867 & MW419185 & MW419248 \\
\hline L. theobromae & CPC 27882 & Citrus sinensis, trunk & Malta & MW413868 & MW419186 & MW419249 \\
\hline L. theobromae & CPC 27883 & Citrus sinensis, trunk & Malta & MW413869 & MW419187 & MW419250 \\
\hline L. theobromae & CPC 27884 & Citrus sinensis, trunk & Malta & MW413870 & MW419188 & MW419251 \\
\hline L. theobromae & CPC 27885 & Citrus sinensis, trunk & Malta & MW413871 & MW419189 & MW419252 \\
\hline L. theobromae & CBS 124.13 & - & USA & DQ458890 & DQ458875 & DQ458858 \\
\hline L. theobromae & CBS 164.96, ex-neotype & Fruit along coral reef coast & Papua New Guinea & AY640255 & AY640258 & EU673110 \\
\hline L. venezuelensis & $\begin{array}{c}\text { CBS } 118739=\text { WAC } 12539=\text { CMW 13511 } \\
\text { ex-type }\end{array}$ & Acacia mangium, wood & Venezuela & DQ103547 & EU673305 & EU673129 \\
\hline
\end{tabular}


Table 2. Cont.

\begin{tabular}{|c|c|c|c|c|c|c|}
\hline \multirow{2}{*}{ Species } & \multirow{2}{*}{ Strains ${ }^{1}$} & \multirow{2}{*}{ Host/Substrate } & \multirow{2}{*}{ Country } & \multicolumn{3}{|c|}{ GenBank Numbers $^{2}$} \\
\hline & & & & ITS & TEF1 & TUB2 \\
\hline L. viticola & CBS 128313 = UCD 2553AR, ex-type & $\begin{array}{c}\text { Wedge-shape canker of grapevine cv. } \\
\text { Vignoles (complex hybrid of North } \\
\text { America Vitis species and Vitis } \\
\text { vinifera) }\end{array}$ & USA: Arkansas & HQ288227 & HQ288269 & HQ288306 \\
\hline L. vitis & CBS 124060, ex-type & Vitis vinifera & & KX464148 & KX464642 & KX464917 \\
\hline Neofusicoccum arbuti & $\begin{array}{c}\text { CBS } 117453=\text { CMW 13455, ex-type of } N . \\
\text { andinum }\end{array}$ & Eucalyptus sp. & Venezuela & AY693976 & AY693977 & KX464923 \\
\hline N. arbuti & CBS 116131 = AR 4014, ex-type & Arbutus menziesii, canker & USA: Washington & AY819720 & KF531792 & KF531793 \\
\hline N. australe & CBS 139662 = CMW 6837, ex-type & Acacia sp. & Australia: Victoria & AY339262 & AY339270 & AY339254 \\
\hline N. australe & CMW 6853 & Sequoiadendron & Australia & AY339263 & AY339271 & AY339255 \\
\hline N. brasiliense & CMM 1338, ex-type & Mangifera indica & Brazil & JX513630 & JX513610 & KC794030 \\
\hline N. buxi & CBS 116.75 & Buxus sempervirens, leaf & France & KX464165 & KX464678 & - \\
\hline N. cordaticola & CBS $123634=$ CMW 13992, ex-type & Syzygium cordatum & South Africa & EU821898 & EU821868 & EU821838 \\
\hline N. dianense & CSF6075 = CGMCC3.20082, ex-type & $\begin{array}{c}\text { Eucalyptus urophylla } \times \text { E. grandis tree, } \\
\text { twigs }\end{array}$ & China & MT028605 & MT028771 & MT028937 \\
\hline N. eucalypticola & CBS $115679=$ CMW 6539, ex-type & Eucalyptus grandis & Australia & AY615141 & AY615133 & AY615125 \\
\hline N. eucalyptorum & CBS $115791=$ CMW $10125=$ BOT 24 & Eucalyptus grandis & South Africa & AF283686 & AY236891 & AY236920 \\
\hline N. grevilleae & CBS 129518, ex-type & Grevillea aurea & Australia & JF951137 & - & - \\
\hline N. hellenicum & CERC 1947 = CFCC 50067, ex-type & Pistacia vera cultivar Aegina & Greece & KP217053 & KP217061 & KP217069 \\
\hline N. hongkongense & CERC2973 = CGMCC3.18749, ex-type & Araucaria cunninghamii & China & KX278052 & KX278157 & KX278261 \\
\hline N. illicii & CGMCC3.18310, ex-type & Illicium verum & China & KY350149 & - & KY350155 \\
\hline N. kwambonambiense & CBS $123639=$ CMW 14023, ex-type & Syzygium cordatum & South Africa & EU821900 & EU821870 & EU821840 \\
\hline N. lumnitzerae & CBS $139674=$ CMW 41469, ex-type & Lumnitzera racemosa & South Africa & KP860881 & KP860724 & KP860801 \\
\hline
\end{tabular}


Table 2. Cont.

\begin{tabular}{|c|c|c|c|c|c|c|}
\hline \multirow{2}{*}{ Species } & \multirow{2}{*}{ Strains $^{1}$} & \multirow{2}{*}{ Host/Substrate } & \multirow{2}{*}{ Country } & \multicolumn{3}{|c|}{ GenBank Numbers $^{2}$} \\
\hline & & & & ITS & TEF1 & TUB2 \\
\hline N. luteum & CPC 27961 & Citrus limon, twig & Portugal & MW413872 & MW419190 & MW419253 \\
\hline N. luteum & СРС 27962 & Citrus limon, twig & Portugal & MW413873 & MW419191 & MW419254 \\
\hline N. luteum & CBS $110497=$ CPC $4594=$ CAP 037 & Vitis vinifera, grape & Portugal & EU673311 & EU673277 & EU673092 \\
\hline N. luteum & CBS 110299 = LM 926 = CAP 002, ex-type & Vitis vinifera, cane & Portugal & AY259091 & KX464688 & DQ458848 \\
\hline N. luteum & $\begin{array}{c}\text { CBS } 140738=\text { CMW } 41365, \text { ex-type of } N . \\
\text { mangroviorum }\end{array}$ & Avicennia marina & South Africa & KP860859 & KP860702 & KP860779 \\
\hline N. macroclavatum & $\begin{array}{c}\text { CBS } 118223=\text { CMW } 15955=\text { WAC } 12444, \\
\text { ex-type }\end{array}$ & Eucalyptus globulus, wood & $\begin{array}{l}\text { Australia: Western } \\
\text { Australia }\end{array}$ & DQ093196 & DQ093217 & DQ093206 \\
\hline N. magniconidium & CSF5876 = CGMCC3.20077, ex-type & $\begin{array}{c}\text { Eucalyptus urophylla } \times \text { E. grandis tree, } \\
\text { twigs }\end{array}$ & China & МT028612 & MT028778 & MT028944 \\
\hline N. mangiferae & CBS 118531 = CMW 7024 & Mangifera indica & Australia & AY615185 & DQ093221 & AY615173 \\
\hline N. mediterraneum & CBS $121718=$ CPC 13137, ex-type & Eucalyptus sp., branches and leaves & Greece & GU251176 & - & - \\
\hline N. mediterraneum & $\begin{array}{c}\text { CBS } 113083=\text { CPC } 5263, \text { ex-type of } N . \\
\text { pistaciarum }\end{array}$ & Pistacia vera & USA: California & KX464186 & KX464712 & KX464998 \\
\hline N. mediterraneum & $\begin{aligned} \text { CBS } 113089= & \text { CPC } 5274, \text { ex-type of } N . \\
& \text { pistaciicola }\end{aligned}$ & Pistacia vera & USA: California & KX464199 & KX464727 & KX465014 \\
\hline N. mediterraneum & CРC 27931 & Citrus limon, twig & Portugal & MW413874 & MW419192 & MW419255 \\
\hline N. mediterraneum & СРC 27932 & Citrus limon, twig & Portugal & MW413875 & MW419193 & MW419256 \\
\hline N. mediterraneum & CPC 27935 & Citrus limon, twig & Portugal & MW413876 & MW419194 & MW419257 \\
\hline N. mediterraneum & СРC 27936 & Citrus limon, twig & Portugal & MW413877 & MW419195 & MW419258 \\
\hline N. microconidium & CERC3497 = CGMCC3.18750, ex-type & Eucalyptus urophylla $\times$ E. grandis tree & China & KX278053 & KX278158 & KX278262 \\
\hline N. nonquaesitum & CBS 126655 = L3IE1 = PD484, ex-type & $\begin{array}{l}\text { Umbellularia californica, cankered } \\
\text { branch }\end{array}$ & USA: California & GU251163 & GU251295 & GU251823 \\
\hline N. ningerense & CSF6028 = CGMCC3.20078, ex-type & $\begin{array}{c}\text { Eucalyptus urophylla } \times \text { E. grandis tree, } \\
\text { twigs }\end{array}$ & China & MT028613 & МT028779 & MT028945 \\
\hline
\end{tabular}


Table 2. Cont.

\begin{tabular}{|c|c|c|c|c|c|c|}
\hline \multirow{2}{*}{ Species } & \multirow{2}{*}{ Strains $^{1}$} & \multirow{2}{*}{ Host/Substrate } & \multirow{2}{*}{ Country } & \multicolumn{3}{|c|}{ GenBank Numbers $^{2}$} \\
\hline & & & & ITS & TEF1 & TUB2 \\
\hline N. occulatum & CBS 128008 = MUCC 227, ex-type & Eucalyptus grandis hybrid & Australia: Queensland & EU301030 & EU339509 & EU339472 \\
\hline N. pandanicola & $\begin{array}{c}\text { MFLUCC 17-2270 = KUMCC 17-0184, } \\
\text { ex-type }\end{array}$ & Pandanus sp. & China & MH275072 & - & - \\
\hline N. parviconidium & CSF5667 = CGMCC3.20074, ex-type & Eucalyptus tree, twigs & China & MT028615 & MT028781 & MT028947 \\
\hline N. parvum & $\begin{array}{c}\text { CBS } 138823=\text { ICMP } 8003=\text { CMW 9081 = } \\
\text { BOT2487 = ATCC 58191, ex-type }\end{array}$ & Populus nigra, bark of dead twig & New Zealand & AY236943 & AY236888 & AY236917 \\
\hline N. paroum & СРС 26119 & $\begin{array}{c}\text { Citrus sinensisx Poncirus trifoliata, } \\
\text { trunk }\end{array}$ & Italy & MW413878 & MW419196 & MW419259 \\
\hline N. paroum & СРС 26120 & $\begin{array}{c}\text { Citrus sinensisx Poncirus trifoliata, } \\
\text { trunk }\end{array}$ & Italy & MW413879 & MW419197 & MW419260 \\
\hline N. parvum & СРС 26121 & $\begin{array}{c}\text { Citrus sinensisx Poncirus trifoliata, } \\
\text { trunk }\end{array}$ & Italy & MW413880 & MW419198 & MW419261 \\
\hline N. paroum & СРС 26122 & $\begin{array}{c}\begin{array}{c}\text { Citrus sinensisx Poncirus trifoliata, } \\
\text { trunk }\end{array}\end{array}$ & Italy & MW413881 & MW419199 & MW419262 \\
\hline N. paroum & СРС 26124 & $\begin{array}{c}\begin{array}{c}\text { Citrus sinensisx Poncirus trifoliata, } \\
\text { trunk }\end{array}\end{array}$ & Italy & MW413882 & MW419200 & MW419263 \\
\hline N. paroum & СРС 26126 & $\begin{array}{c}\begin{array}{c}\text { Citrus sinensisx Poncirus trifoliata, } \\
\text { trunk }\end{array} \\
\end{array}$ & Italy & MW413883 & MW419201 & MW419264 \\
\hline N. parvum & СРС 26127 & $\begin{array}{c}\text { Citrus sinensisx Poncirus trifoliata, } \\
\text { trunk }\end{array}$ & Italy & MW413884 & MW419202 & MW419265 \\
\hline N. parvum & СРС 26128 & $\begin{array}{c}\begin{array}{c}\text { Citrus sinensisx Poncirus trifoliata, } \\
\text { trunk }\end{array} \\
\end{array}$ & Italy & MW413885 & MW419203 & MW419266 \\
\hline N. paroum & СРС 26129 & $\begin{array}{c}\begin{array}{c}\text { Citrus sinensisx Poncirus trifoliata, } \\
\text { trunk }\end{array} \\
\end{array}$ & Italy & MW413886 & MW419204 & MW419267 \\
\hline N.parvum & СРС 26130 & $\begin{array}{c}\begin{array}{c}\text { Citrus sinensisx Poncirus trifoliata, } \\
\text { trunk }\end{array} \\
\end{array}$ & Italy & MW413887 & MW419205 & MW419268 \\
\hline N. paroum & СРС 27866 & Citrus limon, branch & Malta & MW413888 & MW419206 & MW419269 \\
\hline N. paroum & СРС 27867 & Citrus limon, branch & Malta & MW413889 & MW419207 & MW419270 \\
\hline
\end{tabular}


Table 2. Cont.

\begin{tabular}{|c|c|c|c|c|c|c|}
\hline \multirow{2}{*}{ Species } & \multirow{2}{*}{ Strains $^{1}$} & \multirow{2}{*}{ Host/Substrate } & \multirow{2}{*}{ Country } & \multicolumn{3}{|c|}{ GenBank Numbers $^{2}$} \\
\hline & & & & ITS & TEF1 & TUB2 \\
\hline N. paroum & CPC 27868 & Citrus limon, branch & Malta & MW413890 & MW419208 & MW419271 \\
\hline N. parvum & CPC 28173 & Microcitrus australasica, twig & Italy & MW413891 & MW419209 & MW419272 \\
\hline N. paroum & CPC 28175 & Microcitrus australasica, twig & Italy & MW413892 & MW419210 & MW419273 \\
\hline N. paroum & СРC 28177 & Microcitrus australasica, twig & Italy & MW413893 & MW419211 & MW419274 \\
\hline N. parvum & CBS 110301 = CAP 074 & Vitis vinifera & Portugal & AY259098 & AY573221 & EU673095 \\
\hline N. parvum & MFLUCC 15-09002, ex-type of N. italicum & Vitis vinifera & Italy & KY856755 & KY856754 & - \\
\hline N. parvum & $\begin{array}{c}\text { CBS } 137504=\text { ALG1, ex-type of } N . \\
\text { algeriense }\end{array}$ & Vitis vinifera, branches & Algeria & KJ657702 & KJ657721 & - \\
\hline N. pennatisporum & WAC 13153 = MUCC 510, ex-type & Allocasuarina fraseriana, healthy stem & $\begin{array}{l}\text { Australia: Western } \\
\text { Australia }\end{array}$ & EF591925 & EF591976 & EF591959 \\
\hline N. pistaciae & $\begin{array}{c}\text { CBS 595.76, ex-isotype of Camarosporium } \\
\text { pistaciae }\end{array}$ & Pistacia vera, fruits & Greece & KX464163 & KX464676 & KX464953 \\
\hline N. protearum & CBS 114176 = CPC 1775 = JT 189, ex-type & $\begin{array}{c}\text { Leucadendron salignum } \times \text { L. laureolum } \\
\text { cv. Silvan Red, stems }\end{array}$ & South Africa & AF452539 & KX464720 & KX465006 \\
\hline N. ribis & CBS 115475 = CMW 7772, ex-type & Ribes vulgare & USA & AY236935 & AY236877 & AY236906 \\
\hline N. ribis & $\begin{aligned} \text { CBS } 124924= & \text { CMW 28363, ex-type of } N . \\
& \text { batangarum }\end{aligned}$ & Terminalia catappa, healthy branches & Cameroon & FJ900607 & FJ900653 & FJ900634 \\
\hline N. ribis & $\begin{aligned} \text { CBS } 123645= & \text { CMW 14058, ex-type of } N . \\
& \text { umdonicola }\end{aligned}$ & Syzygium cordatum & South Africa & EU821904 & EU821874 & EU821844 \\
\hline N. sinense & CGMCC3.18315, ex-type & Unknown woody plant & China & KY350148 & KY817755 & KY350154 \\
\hline N. sinoeucalypti & CERC2005 = CGMCC3.18752, ex-type & Eucalyptus urophylla $\times$ E. grandis tree & China & KX278061 & KX278166 & KX278270 \\
\hline N. stellenboschiana & $\begin{array}{c}\text { CBS } 110864=\text { STE-U } 4598=\text { CPC } 4598, \\
\text { ex-type }\end{array}$ & Vitis vinifera & South Africa & AY343407 & AY343348 & KX465047 \\
\hline N. terminaliae & CBS $125264=$ CMW 26683 & Terminalia sericea & South Africa & GQ471804 & GQ471782 & KX465053 \\
\hline N. ursorum & CBS 122811 = CMW 24480, ex-type & Eucalyptus sp. & South Africa & FJ752746 & FJ752709 & KX465056 \\
\hline N. variabile & CMW 37739, ex-type & Mimusops caffra & South Africa & MH558608 & - & MH569153 \\
\hline
\end{tabular}


Table 2. Cont.

\begin{tabular}{|c|c|c|c|c|c|c|}
\hline \multirow{2}{*}{ Species } & \multirow{2}{*}{ Strains ${ }^{1}$} & \multirow{2}{*}{ Host/Substrate } & \multirow{2}{*}{ Country } & \multicolumn{3}{|c|}{ GenBank Numbers $^{2}$} \\
\hline & & & & ITS & TEF1 & TUB2 \\
\hline N. viticlavatum & CBS $112878=$ CPC $5044=$ JM 86, ex-type & Vitis vinifera & South Africa & AY343381 & AY343342 & KX465058 \\
\hline N. vitifusiforme & $\begin{aligned} \text { CBS } 120081= & \text { CPC } 12925, \text { ex-type of } N . \\
& \text { corticosae }\end{aligned}$ & Eucalyptus corticosa, leaves & $\begin{array}{l}\text { Australia: New South } \\
\text { Wales }\end{array}$ & DQ923533 & KX464682 & KX464958 \\
\hline N. vitifusiforme & $\begin{array}{c}\text { CBS } 121112=\text { CPC } 5912, \text { ex-type of } N . \\
\text { pruni }\end{array}$ & Prunus salicina & South Africa & EF445349 & EF445391 & KX465016 \\
\hline N. yunnanense & CSF6142 = CGMCC3.20083, ex-type & Eucalyptus globulus, twigs & China & MT028667 & MT028833 & MT028999 \\
\hline
\end{tabular}

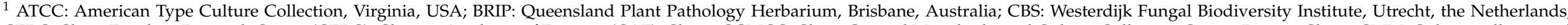

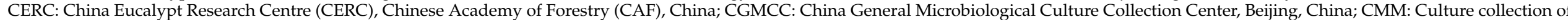

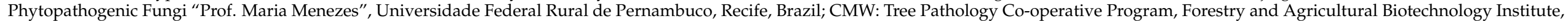

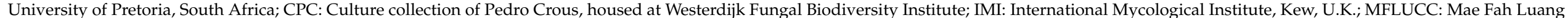

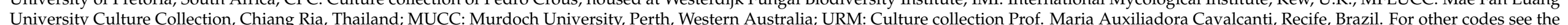
GenBank accession numbers. ${ }^{2}$ ITS: internal transcribed spacers and intervening 5.8S nrDNA; TEF1: partial translation elongation factor 1-alpha gene; TUB2: partial $\beta$-tubulin gene. 


\subsection{Phylogenetic Analyses}

A combined multi-marker (ITS, TEF1, and TUB2) phylogenetic tree was inferred for each genus (Diplodia, Dothiorella, Lasiodiplodia, and Neofusicoccum) obtained in this study (Figures 2-5). The best nucleotide models for the Bayesian Inference analysis of each dataset were as follows: SYM (symmetrical model) + I (proportion of invariable sites) $+\mathrm{G}$ (gamma distribution) (Diplodia, Dothiorella, Lasiodiplodia, and Neofusicoccum) for ITS; GTR (generalized time-reversible model) + G (Diplodia, Dothiorella and Neofusicoccum) and HKY (Hasegawa-Kishino-Yano) + I + G (Lasiodiplodia) for TEF1 and GTR + G (Diplodia, Lasiodiplodia and Neofusicoccum) and GTR + I + G (Dothiorella) for TUB2. The Diplodia phylogenetic analysis revealed the isolates as belonging to Di. pseudoseriata (15 isolates, BPP = 1 and ML-BS $=100)$, Di. seriata (9 isolates, BPP $=1$ and ML-BS $=95$ ), Di. olivarum ( 2 isolates, Bayesian posterior probabilities (BPP) $=1$ and maximum likelihood bootstrapped (ML-BS) = 99), and Di. mutila (1 isolate, BPP = 0.99 and ML-BS = 87) (Figure 2). The Dothiorella phylogeny (Figure 3) grouped the isolates together within Do. viticola (9 isolates, BPP $=1$ and ML-BS = 99). The Lasiodiplodia phylogenetic analysis placed five isolates as L. theobromae (BPP $=1$ and ML-BS = 98) (Figure 4). The Neofusicoccum phylogeny (Figure 5) grouped sequences from our isolates as belonging to N. luteum ( 2 isolates, BPP $=1$ and ML-BS $=94$ ), $N$. parvum (16 isolates) and N. mediterraneum (4 isolates, $\mathrm{BPP}=1$ and ML-BS $=98$ ).

\subsection{Occurrence of Botryosphaeriaceae among Countries and Citrus Species}

Among countries, Do. viticola was found in Greece, Italy, Portugal, and Spain; N. paroum in Italy and Malta, and Di. pseudoseriata in Portugal and Spain. In addition, Di. mutila and Di. seriata were exclusively isolated in Greece and Spain, respectively; $L$. theobromae and Di. olivarum were only found in Malta, and N. luteum and N. mediterraneum were exclusively found in Portugal. Based on the citrus species, N. parvum $(25.4 \%)$ and Di. pseudoseriata $(23.8 \%)$ were the most frequently detected Botryosphaeriaceae spp. on C. sinensis $\times$ P. trifoliata, C. limon, C. reticulata, C. sinensis, and/or M. australasica; Di. seriata (on C. reticulata and C. sinensis); and Do. viticola (on C. aurantium and C. sinensis) had an equal percentage of frequency (14.3\%); Di. mutila (exclusively found on C. sinensis), $N$. luteum and N. mediterraneum (only found on C. limon) and Di. olivarum and L. theobromae (exclusively found on $C$. sinensis) had low frequency values varying from $1.6 \%$ to $7.9 \%$.

\subsection{Pathogenicity Tests}

All isolates caused lesions on wood of inoculated plants $60 \mathrm{~d}$ after inoculation (Figure 6) and the fungi were successfully re-isolated. No lesions were observed on control plants. The frequency of re-isolation was between $90 \%$ and $95 \%$. The identities of the respective inoculated and re-isolated species were confirmed using culture and molecular analysis as described above, fulfilling Koch's postulates. Lesions and internal discolouration were observed in correspondence to the inoculation points (Figure 7). The inoculated species that showed high aggressiveness on C. sinensis, C. limon, and C. reticulata were Di. seriata, Di. olivarum, L. theobromae, N. mediterraneum, N. luteum, and N. parvum (with mean lesion length (MLL) ranged from 5.25 to $6.96 \mathrm{~cm}$ ). Weak symptoms were caused by $D i$. pseudoseriata, Di. mutila, and Do. viticola on the same species (with MLL ranged from 0.17 to $0.58 \mathrm{~cm}$ ). 


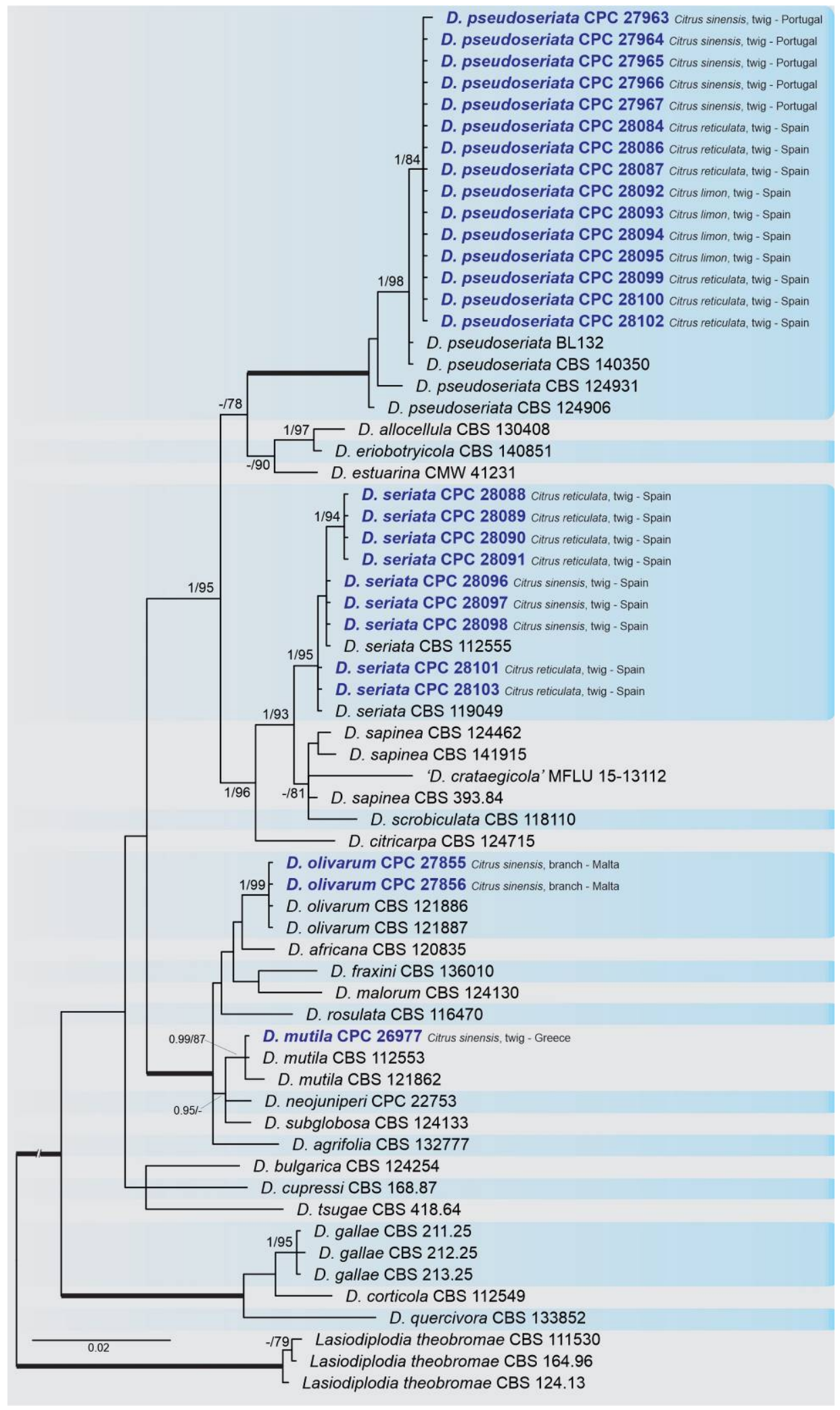

Figure 2. Bayesian inference analysis of Diplodia species using ITS rDNA, TEF1 and TUB2 sequences. Isolates obtained in this study are in bold and blue. Bayesian posterior probability (BPP) and maximum likelihood-bootstrap (ML-BS) values equal or greater than 0.95 and $70 \%$, respectively, are shown near nodes. Thickened branches represent clades with ML-BS $=100 \%$ and a BPP $=1.0$. The tree was rooted to L. theobormae (CBS 111530, CBS 164.96 and CBS 124.13). 


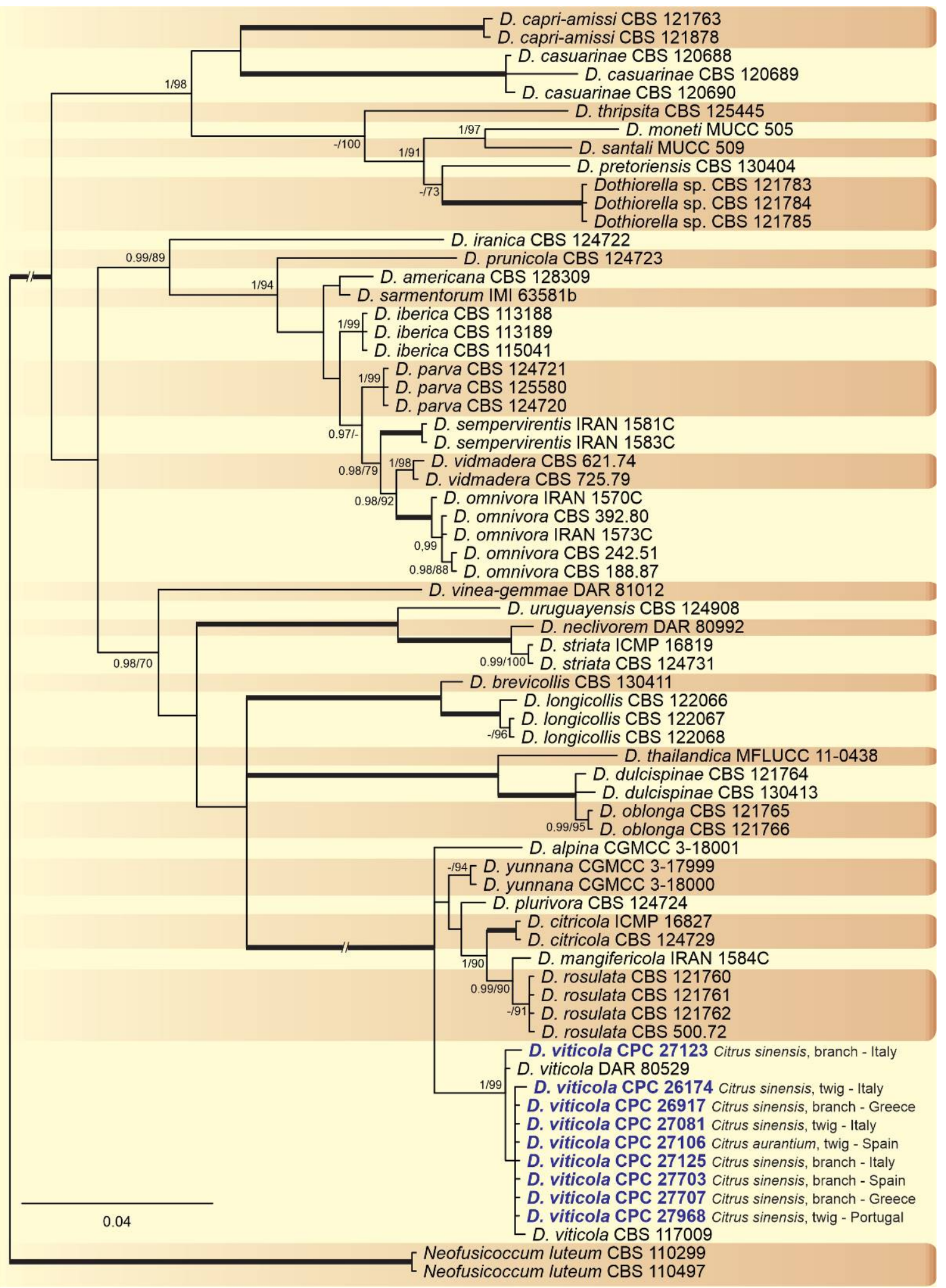

Figure 3. Bayesian inference analysis of Dothiorella species using ITS rDNA, TEF1, and TUB2 sequences. Isolates obtained in this study are in bold and blue. Bayesian posterior probability (BPP) and ML bootstrap (ML-BS) values equal or greater than 0.95 and $70 \%$, respectively, are shown near nodes. Thickened branches represent clades with ML-BS $=100 \%$ and a $\mathrm{BPP}=1.0$. The tree was rooted to N. luteum (CBS 110299 and CBS 110497). 


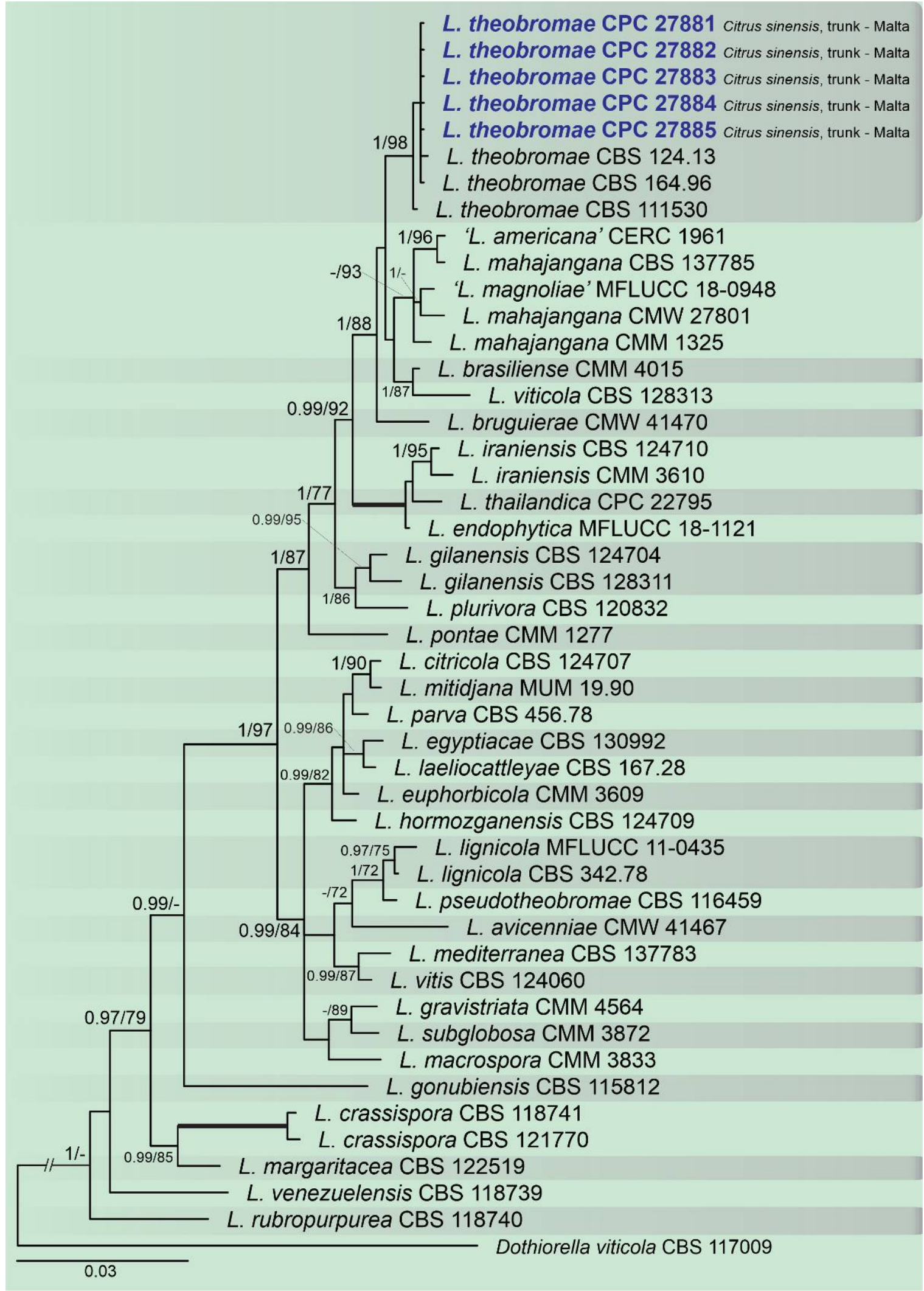

Figure 4. Bayesian inference analysis of Lasiodiplodia species using ITS rDNA, TEF1, and TUB2 sequences. Isolates obtained in this study are in bold and blue. Bayesian posterior probability (BPP) and ML bootstrap (ML-BS) values equal or greater than 0.95 and 70\%, respectively, are shown near nodes. Thickened branches represent clades with ML-BS $=100 \%$ and a $\mathrm{BPP}=1.0$. The tree was rooted to Do. viticola (CBS 117009). 


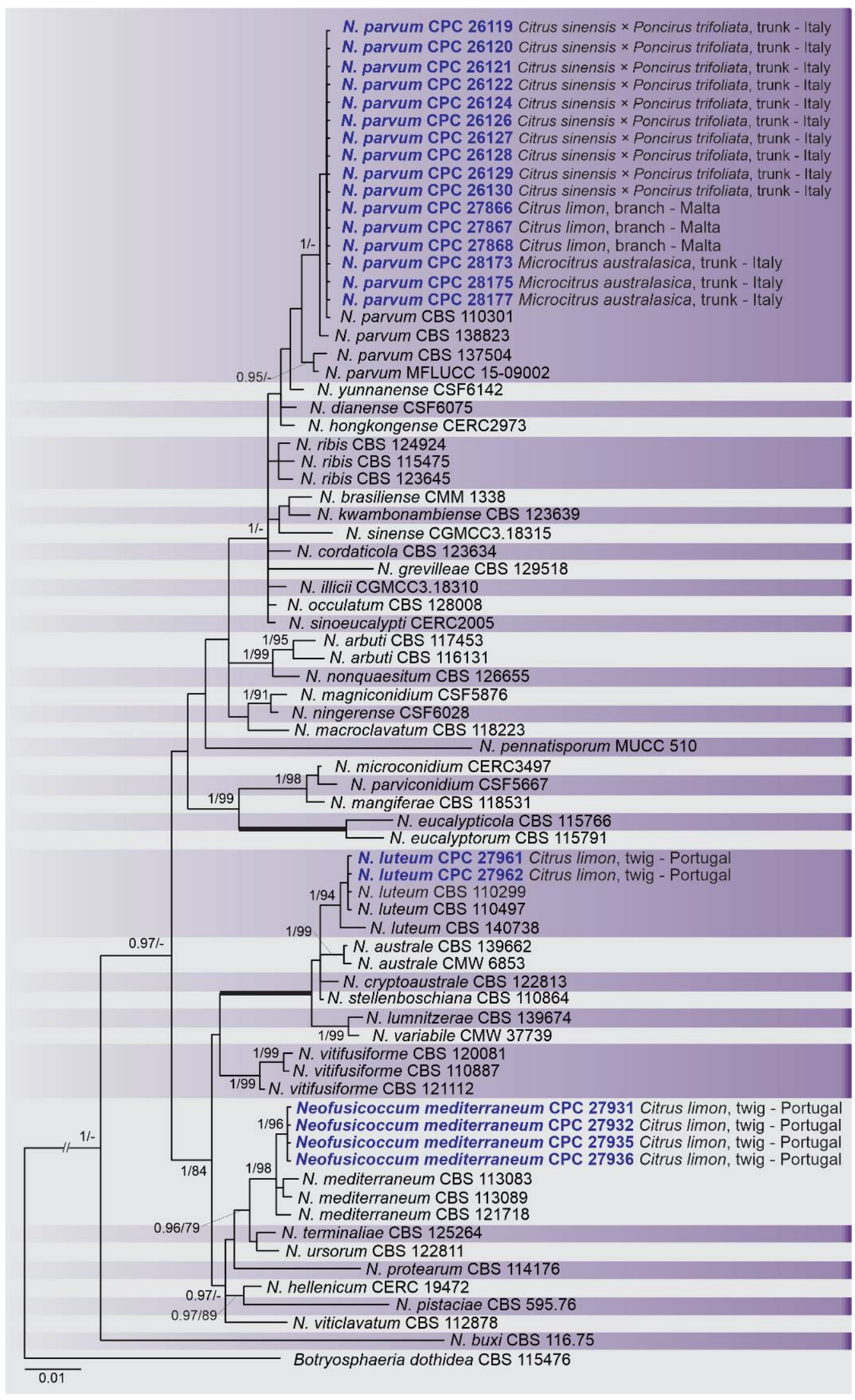

Figure 5. Bayesian inference analysis of species Neofusicoccum using ITS rDNA, TEF1, and TUB2 sequences. Isolates obtained in this study are in bold and blue. Bayesian posterior probability (BPP) and ML bootstrap (ML-BS) values equal or greater than 0.95 and $70 \%$, respectively, are shown near nodes. Thickened branches represent clades with ML-BS $=100 \%$ and a BPP $=1.0$. The tree was rooted to B. dothidea (CBS 115476). 


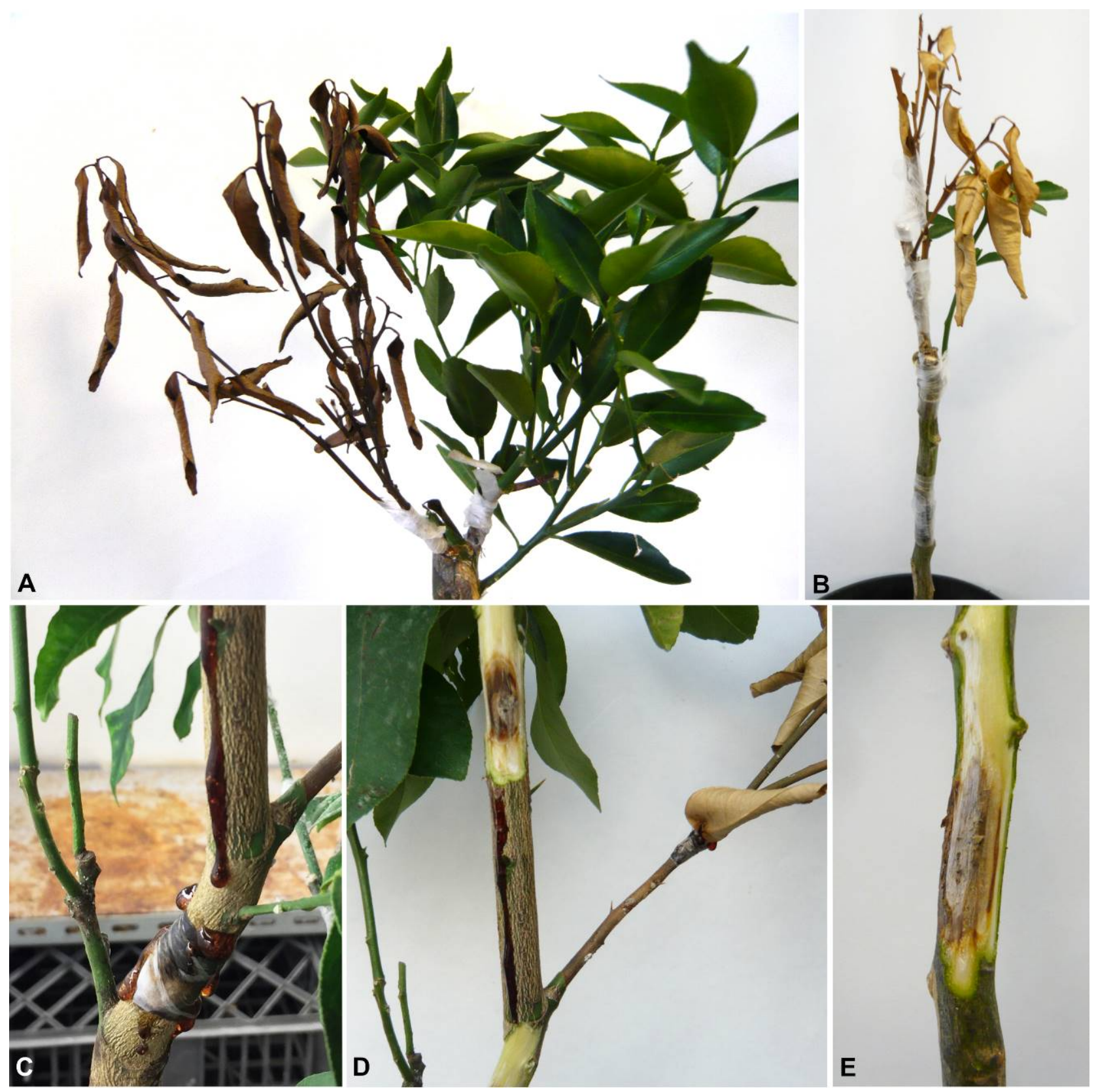

Figure 6. Pathogenicity tests of selected Botryosphaeriacae isolates on citrus plants $60 \mathrm{~d}$ after inoculation. (A,B) Shoot blight of $C$. reticulata and C. sinensis plants inoculated with N. mediterraneum. (C) Internal lesion with abundant gummosis of $C$. sinensis plant caused by N. paroum. (D,E) Internal discoloration of C. sinensis and C. reticulata twigs inoculated with L. theobromae. 


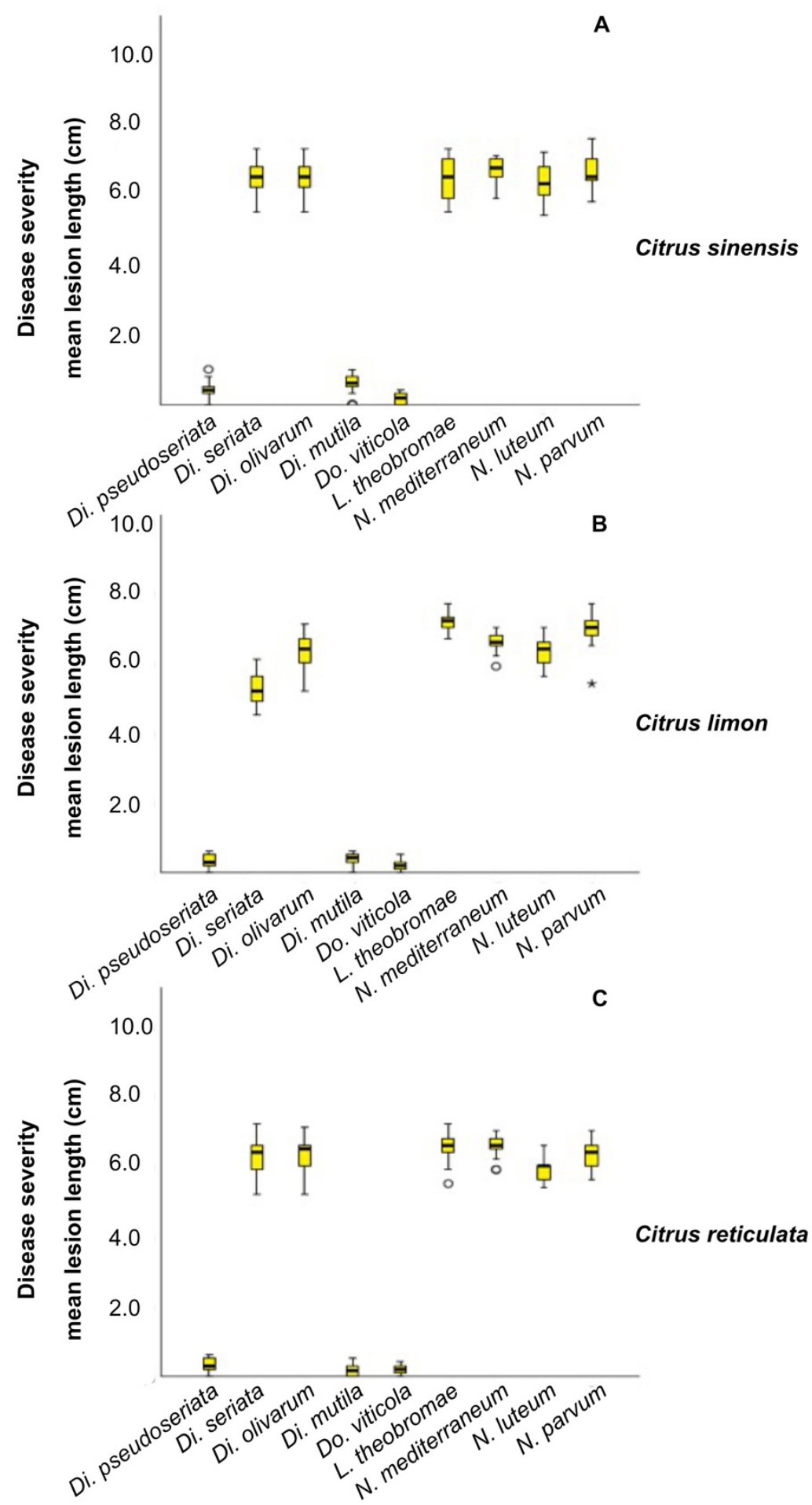

Figure 7. Box plot showing the results of the pathogenicity tests. Boxes represent the interquartile range, while the horizontal line within each box indicates the average value. The Kruskal-Wallis test was carried out to compare the mean lesion lengths $(\mathrm{cm})$ from inoculation with nine Botryosphaeriaceae representative isolates on C. sinensis (A), C. limon (B) and C. reticulata (C). $p<0.05$ was taken to indicate a significant difference. ${ }^{\circ}$ : Outliers. ${ }^{*}$ : Extreme values.

For each tested host species, the pairwise comparison, obtained from the KruskalWallis test, showed significant differences $(p<0.05)$ between the species Di. seriata, Di. olivarum, L. theobromae, N. mediterraneum, N. luteum, and N. parvum and the remaining pathogens Di. pseudoseriata, Di. mutila and Do. viticola (Supplementary Tables S1-S3). 
No significant differences were observed within the group composed by Di. seriata, Di. olivarum, L. theobromae, N. mediterraneum, N. luteum, and N. parvum. Moreover, N. parvum revealed to be highly aggressive on $M$. australasica and C. sinensis $\times$ P. trifoliata with similar level of aggressiveness (Figure 8). The tested strain developed a MLL $=7.83 \mathrm{~cm}$ on $M$. australasica and a MLL $=7.45 \mathrm{~cm}$ on $C$. sinensis $\times$ P. trifoliata.

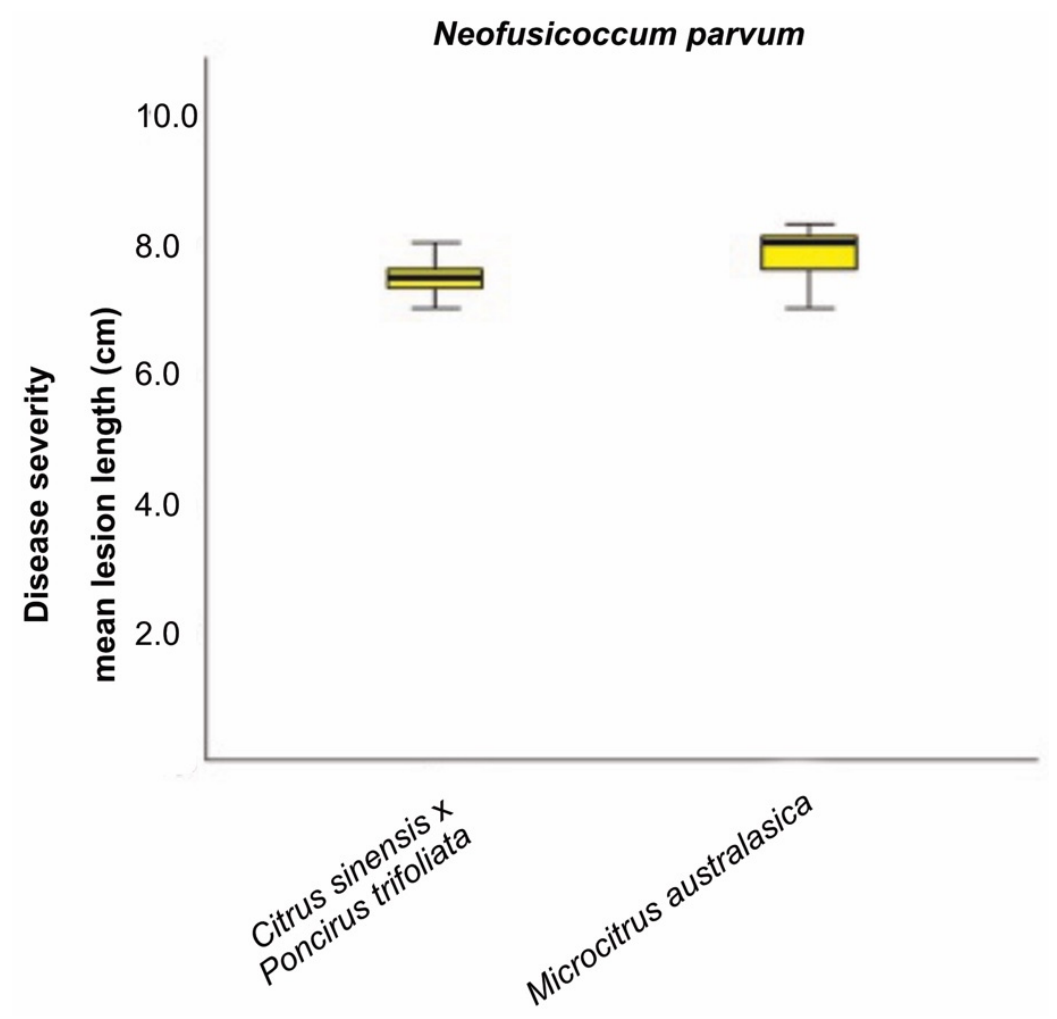

Figure 8. The Kruskal-Wallis test was carried out to compare the mean lesion lengths (cm) from inoculation with one $N$. paroum representative isolate on C. sinensis $\times$ P. trifoliata and M. australasica. Significant difference was accepted for $p<0.05$.

\section{Discussion}

Several Botryosphaeriaceae spp. have been detected in association with citrus cankers worldwide. Diplodia seriata, Di. mutila, Do. iberica, Do. viticola, L. parva, N. australe, N. luteum, N. mediterraneum, N. paroum, and Ne. dimidiatum have been recovered from necrotic tissues of branch canker and rootstock citrus samples in California [13,31,36]. Recently, Di. citricarpa was described for a fungus on twigs of Citrus sp. in Iran [16] and L. mitidjana was introduced for a fungus causing branch canker and dieback of C. sinensis in Algeria [33]. Botryosphaeriaceae spp. causing disease on citrus are known in European countries, where N. parvum and Ne. dimidiatum were reported on C. reticulata in Greece and on C. sinensis in Italy, respectively [32,37].

This study represents the first large survey aimed at studying the occurrence, genetic diversity, and pathogenicity of Botryosphaeriaceae species associated with symptomatic citrus species of citrus-producing areas in Greece, Italy, Portugal, Malta, and Spain [10,38]. Results obtained during our study have added new information about the pathogenicity of Botryosphaeriaceae spp. in citrus-producing areas of these European countries. Symptomatic plants were observed during fieldwork in all the citrus orchards and regions investigated and all isolates used in the pathogenicity test caused lesions on wood of inoculated citrus plants. Phylogenetic multi-marker analyses recognized botryosphaeriaceous isolates in four Diplodia species, with Di. pseudoseriata (15 isolates) being the most common; followed by three Neofusicoccum species, with $N$. parvum (16 isolates) as dominant species, Do. viticola (9 isolates), and L. theobromae (5 isolates). All species found in this study, except 
Di. pseudoseriata and Di. olivarum, which are reported for the first time on Citrus spp., have been found in citrus-producing areas of California (USA) [13,31,36].

Diplodia and Neofusicoccum species were dominant in this study. Different species of Neofusicoccum and Diplodia were the most frequently detected pathogens causing gummosis on citrus in California [36] and Di. citricarpa was a new species isolated from Citrus sp. in Iran [16]. Species of Diplodia, Dothiorella, Lasiodiplodia, and Neofusicoccum detected in our study are widely reported as pathogens of other host plants in Algeria and Tunisia [39,40], Australia [41], Brazil [42], China [43,44], Chile [45], Italy, Portugal [39,46-48], South Africa [49], and the USA [13,31,36]. The results obtained in our study provide valuable information related to the richness, occurrence, and pathogenicity of Botryosphaeriaceae species in association with citrus species. This study is also the first major survey for Botryosphaeriaceae species associated with symptomatic citrus species in citrus-producing areas of five European countries, providing essential information for future monitoring. Moreover, while previous reports of canker diseases of citrus were based exclusively on morphological observations, the current study aimed to investigate the fungi affecting the major citrus production areas in Europe by large-scale sampling, morphology, and DNA phylogeny. The information achieved with this study about Botryosphaeriaceae population and citrus canker etiology provide fundamental knowledge to start further studies aimed to improve the disease management.

\section{Materials and Methods}

\subsection{Field Sampling and Fungal Isolation}

During 2015 and 2016 more than 90 sites in the most important citrus-producing areas of Europe were investigated. The surveys were conducted in Andalusia, Valencia, and the Balearic Islands (Spain); Apulia, Calabria, Sicily, and Aeolian Islands (Italy); Algarve (Portugal); Arta, Crete, Missolonghi, and Nafplio (Greece); Malta and Gozo (Malta) [10,38]. Twig, branch and trunk portions showing cankers and dieback were collected. Investigated species of Citrus and allied genera of the Rutaceae family such as Microcitrus included: C. limon, C. reticulata, C. sinensis, M. australasica, and C. sinensis $\times P$. trifoliata.

Wood fragments $(5 \times 5 \mathrm{~mm})$ were collected from the margin between necrotic and healthy tissues. Then, each fragment was disinfected by immersion in $70 \%$ ethanol for $5 \mathrm{~s}, 4 \%$ sodium hypochlorite for $90 \mathrm{~s}$, sterilised distilled water for $60 \mathrm{~s}$ and then dried on sterile filter paper. The fragments were placed into Petri dishes containing malt extract agar (MEA) [50] supplemented with penicillin $(100 \mu \mathrm{g} / \mathrm{mL})$ and streptomycin $(100 \mu \mathrm{g} / \mathrm{mL})$ (MEA-PS) and incubated at $25{ }^{\circ} \mathrm{C}$ until characteristic Botryosphaeriaceae colonies were observed. A second procedure was used with plant material incubated in moist chambers at $20 \pm 3{ }^{\circ} \mathrm{C}$ for up to $10 \mathrm{~d}$ and inspected daily for fungal sporulation. The conidia obtained through both procedures were collected and crushed in a drop of sterile water and then spread over the surface of MEA-PS plates. After $24 \mathrm{~h}$, germinating spores were individually transferred onto MEA plates. The isolates used in this study are maintained in the working collection of Pedro Crous (CPC), housed at the Westerdijk Fungal Biodiversity Institute (CBS), Utrecht, The Netherlands.

The occurrence of botryosphaeriaceous fungi among countries and citrus species was evaluated as the number of isolates from each fungal species against the total number of isolates and expressed as a percentage.

\subsection{DNA Extraction, Polymerase Chain Reaction (PCR) Amplification and Sequencing}

Colonies grown on MEA for 7 days were used to perform total DNA extraction using the Wizard ${ }^{\circledR \circledR}$ Genomic DNA Purification Kit (Promega, Madison, WI, USA) standard protocol. The primer pair ITS4/ITS5 [51] was used to amplify the ITS. The primer sets EF1-728F/EF2 [52,53] and Bt2a/Bt2b [54] were used to amplify partial fragments of the TEF1 and TUB2 genes, respectively. Amplification by PCR was conducted as described by Yang et al. [16]. The PCR products were sequenced in both directions using the BigDye ${ }^{\circledR \mathbb{R}}$ Terminator v. 3.1 Cycle Sequencing Kit (Applied Biosystems Life Technologies, Carlsbad, 
CA, USA), after which amplicons were purified through Sephadex G-50 Fine columns (GE Healthcare, Freiburg, Germany) in MultiScreen HV plates (Millipore, Billerica, MA, USA). Purified sequence reactions were analyzed on an Applied Biosystems 3730xl DNA Analyzer (Life Technologies, Carlsbad, CA, USA). The DNA sequences generated were analyzed and consensus sequences were computed using SeqMan Pro (DNASTAR, Madison, WI, USA). Sequences obtained in this study were deposited in GenBank https:/ /academic.oup.com/ nar/article/49/D1/D92/5983623 (accessed on 30 January 2021) (Table 2).

\subsection{Phylogenetic Analyses}

The phylogenetic analyses included DNA sequences generated in this study along with DNA sequences retrieved from GenBank (Table 2) and represent 124 Botryosphaeriaceae species (Diplodia $=23$; Dothiorella $=31$; Lasiodiplodia $=31$; Neofusicoccum $=39$ ) following recent studies $[16,23,25]$. Alignments were first made using MAFFT v. 7 [55] and manually checked and edited using MEGA v.7 [56]. Maximum Likelihood (ML) and Bayesian Inference (BI) analyses were conducted using RAxML-HPC BlackBox v.8.2.8 [57] and MrBayes v.3.2.7a on XSEDE, respectively, at the CIPRES Science Gateway. The best nucleotide models for the BI analysis were calculated using MrModelTest v.2.3 [58] while GTR + I + G was used for ML analysis. Clade stability of the ML phylogeny was assessed with 1000 bootstrap replicates. The BI analysis lasted for one million generations, a burning value of $25 \%$ and chains were sampled every 1000 generations. Values of ML bootstrap (ML-BS) and BI posterior probability (BPP) equal or greater than $70 \%$ and 0.95 , respectively, were considered significant. Individual gene phylogenies were visually inspected and compared for topological incongruences before combining into a multi-marker sequence alignment. The combined alignments used to perform the phylogenetic inferences were deposited in TreeBASE (study ID S27709).

\subsection{Pathogenicity Tests}

Pathogenicity tests with nine Botryosphaeriaceae species isolated from the European citrus samples were performed to satisfy Koch's postulates.

One isolate of Di. pseudoseriata (CPC 28084), Di. seriata (CPC 28091), Di. olivarum (CPC 27855), Di. mutila (CPC 26977), Do. viticola (CPC 27125), L. theobromae (CPC 27881), N. mediterraneum (CPC 27931), N. luteum (CPC 27961), and N. parvum (CPC 28175) were respectively inoculated onto potted 2-y-old healthy plants of lemon (C. limon), mandarin (C. reticulata) and sweet orange (C. sinensis). The strain of $N$. parvum was also inoculated onto potted 2-y-old healthy plants of Australasian lime (M. australasica) and Carrizo citrange (C. sinensis $\times$ P. trifoliata).

Three plants for each isolate were inoculated, each having five wounds on twigs made using a sterile blade. Mycelial plugs (5 $\mathrm{mm}$ diam.), taken from the margin of actively growing colonies on MEA, were placed on the wound sites on each plant. An equivalent number of plants and inoculation sites were inoculated with sterile MEA plugs and served as controls. The inoculation sites were covered with Parafilm ${ }^{\circledR \circledR}$ (American National Can, Chicago, IL, USA). The inoculated plants were incubated with a $16 \mathrm{~h}$ photoperiod in a growth chamber at $100 \%$ relative humidity and $25 \pm 1{ }^{\circ} \mathrm{C}$. After 2 months external symptoms were assessed. Twigs were cut and the bark peeled off to check for any internal discolouration and the total, upward and downward lesion length was taken to evaluate the MLL. Small sections $(0.5 \mathrm{~cm})$ of symptomatic tissue from the edge of twig lesions were placed on MEA to re-isolate the fungal species and were identified based on TEF1 sequencing to fulfil Koch's postulates. The experiment was conducted twice and each trial was considered a replicate. Because no normal distribution was observed in the lesion dimension data, the Kruskal-Wallis non-parametric test (at $P=0.05$ ) was performed to determine significant differences among isolates. The data analysis was conducted using SPSS software 26 (IBM Corporate).

Supplementary Materials: The following are available online at https: / www.mdpi.com/2223-7 747/10/3/492/s1, Tables S1-S3. Kruskal-Wallis test results with multiple comparisons for disease 
severity between different Botryosphaeriaceae spp. on artificially inoculated twigs of C. sinensis (Table S1), C. limon (Table S2) and C. reticulata (Table S3).

Author Contributions: Conceptualization, V.G., G.P. and P.W.C.; methodology, J.D.P.B., D.A. and V.G.; software, J.D.P.B., V.G. and D.A.; validation, G.P., J.D.P.B. and V.G.; formal analysis, J.D.P.B., D.A. and V.G.; investigation, V.G. and D.A.; resources, P.W.C.; data curation, J.D.P.B. and V.G.; writingoriginal draft preparation, J.D.P.B. and V.G.; writing—review and editing, G.P., M.L.G. and P.W.C.; supervision, G.P., M.L.G. and P.W.C.; project administration, P.W.C.; funding acquisition, P.W.C. All authors have read and agreed to the published version of the manuscript.

Funding: This research received no external funding.

Institutional Review Board Statement: Not applicable.

Informed Consent Statement: Not applicable.

Acknowledgments: The authors thank G. Gilardi (AGROINNOVA-University of Torino) and A. Azzaro for technical support.

Conflicts of Interest: The authors declare no conflict of interest.

\section{References}

1. FAOSTAT. Food and Agriculture Organization of the United Nations. 2019. Available online: http://www.fao.org/faostat/en/ \#home (accessed on 26 February 2020).

2. Eurostat. Citrus Fruit Statistics; Eurostat: Luxembourg, 2020.

3. Crous, P.W.; Wingfield, M.J. Fungi infecting woody plants: Emerging frontiers. Persoonia 2018, 40, i-iii. [CrossRef]

4. Fawcett, H.S. Citrus Diseases and Their Control; McGraw-Hill: New York, NY, USA, 1936.

5. Huang, F.; Chen, G.Q.; Hou, X.; Fu, Y.S.; Cai, L.; Hyde, K.D.; Li, H.Y. Colletotrichum species associated with cultivated citrus in China. Fungal Divers. 2013, 61, 61-74. [CrossRef]

6. Huang, F.; Hou, X.; Dewdney, M.M.; Fu, Y.S.; Chen, G.Q.; Hyde, K.D.; Li, H. Diaporthe species occurring on citrus in China. Fungal Divers. 2013, 61, 237-250. [CrossRef]

7. Mahadevakumar, S.; Yadav, V.; Tejaswini, G.S.; Sandeep, S.N.; Janardhana, G.R. First report of Phomopsis citri associated with dieback of Citrus lemon in India. Plant Dis. 2014, 98, 1281. [CrossRef]

8. Guarnaccia, V.; Crous, P.W. Species of Diaporthe on Camellia and Citrus in the Azores Islands. Phytopathol. Mediterr. 2018, 57, 307-319.

9. Mayorquin, J.S.; Nouri, M.T.; Peacock, B.B.; Trouillas, F.P.; Douhan, G.W.; Kallsen, C.; Eskalen, A. Identification, Pathogenicity, and Spore Trapping of Colletotrichum karstii Associated with Twig and Shoot Dieback in California. Plant Dis. 2019, 103, 1464-1473. [CrossRef] [PubMed]

10. Guarnaccia, V.; Crous, P.W. Emerging citrus diseases in Europe caused by species of Diaporthe. IMA Fungus 2017, 8, 317-334. [CrossRef] [PubMed]

11. Sandoval-Denis, M.; Guarnaccia, V.; Polizzi, G.; Crous, P.W. Symptomatic Citrus trees reveal a new pathogenic lineage in Fusarium and two new Neocosmospora species. Persoonia 2018, 40, 1-25. [CrossRef] [PubMed]

12. Timmer, L.W.; Garnsey, S.M.; Graham, J.H. Compendium of Citrus Diseases, 2nd ed.; American Phytopathological Society: Saint Paul, MN, USA, 2000.

13. Mayorquin, J.S.; Wang, D.H.; Twizeyimana, M.; Eskalen, A. Identification, distribution, and pathogenicity of Diatrypaceae and Botryosphaeriaceae associated with Citrus branch canker in the southern California desert. Plant Dis. 2016, 100, $2402-2413$. [CrossRef] [PubMed]

14. Espargham, N.; Mohammadi, H.; Gramaje, D.A. Survey of Trunk Disease Pathogens within Citrus Trees in Iran. Plants 2020, 9 , 754. [CrossRef]

15. Phillips, A.J.L.; Alves, A.; Abdollahzadeh, J.; Slippers, B.; Wingfield, M.J.; Groenewald, J.Z.; Crous, P.W. The Botryosphaeriaceae: Genera and species known from culture. Stud. Mycol. 2013, 76, 51-167. [CrossRef]

16. Yang, T.; Groenewald, J.Z.; Cheewangkoon, R.; Jami, F.; Abdollahzadeh, J.; Lombard, L.; Crous, P.W. Families, genera, and species of Botryosphaeriales. Fungal Biol. 2017, 121, 322-346. [CrossRef] [PubMed]

17. Slippers, B.; Wingfield, M.J. Botryosphaeriaceae as endophytes and latent pathogens of woody plants: Diversity, ecology and impact. Fungal Biol. Rev. 2007, 21, 90-106. [CrossRef]

18. Crous, P.W.; Slippers, B.; Wingfield, M.J.; Rheeder, J.; Marasas, W.F.O.; Philips, A.J.L.; Alves, A.; Burgess, T.; Barber, P.; Groenewald, J.Z. Phylogenetic lineages in the Botryosphaeriaceae. Stud. Mycol. 2006, 55, 235-253. [CrossRef] [PubMed]

19. Crous, P.W.; Giraldo, A.; Hawksworth, D.L.; Robert, V.; Kirk, P.M.; Guarro, J.; Robbertse, B.; Schoch, C.L.; Damm, U.; Trakunyingcharoen, T.; et al. The genera of fungi: Fixing the application of type species of generic names. IMA Fungus 2014, 5, 141-160. [CrossRef] [PubMed]

20. Crous, P.W.; Muller, M.M.; Sanchez, R.M.; Giordano, L.; Bianchinotti, M.V.; Anderson, F.E.; Groenewald, J.Z. Resolving Tiarosporella spp. allied to Botryosphaeriaceae and Phacidiaceae. Phytotaxa 2015, 202, 73-93. [CrossRef] 
21. Schoch, C.L.; Shoemaker, R.A.; Seifert, K.A.; Hambleton, S.; Spatafora, J.W.; Crous, P.W. A multigene phylogeny of the Dothideomycetes using four nuclear loci. Mycologia 2006, 98, 1041-1052. [CrossRef]

22. Slippers, B.; Boissin, E.; Phillips, A.J.L.; Groenewald, J.Z.; Lombard, L.; Wingfield, M.J.; Postma, A.; Burgess, T.; Crous, P.W. Phylogenetic lineages in the Botryosphaeriales: A systematic and evolutionary framework. Stud. Mycol. 2013, 76, 31-49. [CrossRef] [PubMed]

23. Phillips, A.J.L.; Hyde, K.D.; Alves, A.; Liu, J. Families in Botryosphaeriales: A phylogenetic, morphological and evolutionary perspective. Fungal Divers. 2019, 94, 1-22. [CrossRef]

24. Wijayawardene, N.N.; Hyde, K.D.; Al-Ani, L.K.T.; Tedersoo, L.; Haelewaters, D.; Rajeshkumar, K.C.; Zhao, R.L.; Aptroot, A.; Leontyev, D.V.; Saxena, R.K.; et al. Outline of Fungi and fungus-like taxa. Mycosphere 2020, 11, 1060-1456. [CrossRef]

25. Zhang, W.; Groenewald, J.Z.; Lombard, L.; Schumacher, R.K.; Phillips, A.J.L.; Crous, P.W. Evaluating species in Botryosphaeriales. Persoonia 2021, 46, 63-115.

26. Mehl, J.W.M.; Slippers, B.; Roux, J.; Wingfield, M.J. Cankers and other diseases caused by the Botryosphaeriaceae. In Infectious Forest Diseases; Gonthier, P., Nicolotti, G., Eds.; CAB International: Boston, MA, USA, 2013; pp. $298-317$.

27. Pour, F.N.; Ferreira, V.; Félix, C.; Serôdio, J.; Alves, A.; Duarte, A.S.; Esteves, A.C. Effect of temperature on the phytotoxicity and cytotoxicity of Botryosphaeriaceae fungi. Fungal Biol. 2020, 124, 571-578. [CrossRef]

28. Guarnaccia, V.; Martino, I.; Tabone, G.; Brondino, L.; Gullino, M.L. Fungal pathogens associated with stem blight and dieback of blueberry in northern Italy. Phytopathol. Mediterr. 2020, 59, 229-245.

29. Marsberg, A.; Kemler, M.; Jami, F.; Nagel, J.H.; Postma-Smidt, A.; Naidoo, S.; Wingfield, M.J.; Crous, P.W.; Spatafora, J.W.; Hesse, C.N.; et al. Botryosphaeria dothidea: A latent pathogen of global importance to woody plant health. Mol. Plant Pathol. 2017, 18, 477-488. [CrossRef] [PubMed]

30. Aiello, D.; Gusella, G.; Fiorenza, A.; Guarnaccia, V.; Polizzi, G. Identification of Neofusicoccum parvum causing canker and twig blight on Ficus carica in Italy. Phytopathol. Mediterr. 2020, 59, 213-218.

31. Adesemoye, A.O.; Eskalen, A. First Report of Spencermartinsia viticola, Neofusicoccum australe, and N. parvum causing branch canker of citrus in California. Plant Dis. 2011, 95, 770. [CrossRef] [PubMed]

32. Polizzi, G.; Aiello, D.; Vitale, A.; Giuffrida, F.; Groenewald, J.; Crous, P.W. First report of shoot blight, canker, and gummosis caused by Neoscytalidium dimidiatum on citrus in Italy. Plant Dis. 2009, 93, 1215. [CrossRef]

33. Berraf-Tebbal, A.; Mahamedi, A.E.; Aigoun-Mouhous, W.; Špetík, M.; Čechová, J.; Pokluda, R.; Baránek, M.; Eichmeier, A.; Alves, A. Lasiodiplodia mitidjana sp. nov. and other Botryosphaeriaceae species causing branch canker and dieback of Citrus sinensis in Algeria. PLoS ONE 2020, 15, e0232448. [CrossRef]

34. Abdollahzadeh, J.; Javadi, A.; Goltapeh, E.M.; Zare, R.; Phillips, A.J. Phylogeny and morphology of four new species of Lasiodiplodia from Iran. Persoonia 2010, 25, 1-10. [CrossRef]

35. Hamrouni, N.; Nouri, M.; Trouillas, F.; Said, A.; Sadfi-Zouaoui, N.; Hajlaoui, M. Dothiorella gummosis caused by Dothiorella viticola, first record from citrus in Tunisia. New Dis. Rep. 2018, 38, 10. [CrossRef]

36. Adesemoye, A.O.; Mayorquin, J.S.; Wang, D.H.; Twizeyimana, M.; Lynch, S.C.; Eskalen, A. Identification of species of Botryosphaeriaceae causing bot gummosis in citrus in California. Plant Dis. 2014, 98, 55-61. [CrossRef] [PubMed]

37. Vakalounakis, D.J.; Ntougias, S.; Kavroulakis, N.; Protopapadakis, E. Neofusicoccum parvum and Diaporthe foeniculina associated with twig and shoot blight and branch canker of citrus in Greece. J. Phytopathol. 2019, 167, 527-537. [CrossRef]

38. Guarnaccia, V.; Groenewald, J.Z.; Li, H.; Glienke, C.; Carstens, E.; Hattingh, V.; Crous, P.W. First report of Phyllosticta citricarpa and description of two new species, P. paracapitalensis and P. paracitricarpa, from citrus in Europe. Stud. Mycol. 2017, 87, 161-185. [CrossRef]

39. Linaldeddu, B.T.; Deidda, A.; Scanu, B.; Franceschini, A.; Serra, S.; Berraf-Tebbal, A.; Boutiti, M.Z.; Jamâa, M.L.B.; Phillips, A.J.L. Diversity of Botryosphaeriaceae species associated with grapevine and other woody hosts in Italy, Algeria and Tunisia, with descriptions of Lasiodiplodia exigua and Lasiodiplodia mediterranea sp. nov. Fungal Divers. 2015, 71, 201-214. [CrossRef]

40. Mahamedi, A.E.; Phillips, A.J.L.; Lopes, A.; Djellid, Y.; Arkam, M.; Eichmeier, A.; Zitouni, A.; Alves, A.; Berraf-Tebbal, A. Diversity, distribution and host association of Botryosphaeriaceae species causing oak decline across different forest ecosystems in Algeria. Eur. J. Plant Pathol. 2020, 158, 745-765. [CrossRef]

41. Burgess, T.I.; Tan, Y.P.; Garnas, J.; Edwards, J.; Scarlett, K.A.; Shuttleworth, L.A.; Daniel, R.; Dann, E.K.; Parkinson, L.E.; Dinh, Q. Current status of the Botryosphaeriaceae in Australia. Australas. Plant Pathol. 2018, 48, 35-44. [CrossRef]

42. Machado, A.R.; Custódio, F.A.; Cabral, P.G.C.; Capucho, A.S.; Pereira, O.L. Botryosphaeriaceae species causing dieback on Annonaceae in Brazil. Plant Pathol. 2019, 68, 1394-1406. [CrossRef]

43. Xu, C.; Zhang, H.; Zhou, Z.; Hu, T.; Wang, S.; Wang, Y.; Cao, K. Identification and distribution of Botryosphaeriaceae species associated with blueberry stem blight in China. Eur. J. Plant Pathol. 2015, 143, 737-752. [CrossRef]

44. Li, G.; Slippers, B.; Wingfield, M.J.; Chen, S. Variation in Botryosphaeriaceae from Eucalyptus plantations in YunNan Province in southwestern China across a climatic gradient. IMA Fungus 2020, 11, 22. [CrossRef]

45. Valencia, A.L.; Pilar, M.; Gil, B.A.; Latorre, I.; Rosales, M. Characterization and Pathogenicity of Botryosphaeriaceae Species Obtained from Avocado Trees with Branch Canker and Dieback and from Avocado Fruit with Stem End Rot in Chile. Plant Dis. 2019, 103, 996-1005. [CrossRef]

46. Gusella, G.; Aiello, D.; Polizzi, G. First report of leaf and twig blight of Indian hawthorn (Rhaphiolepis indica) caused by Neofusicoccum parvum in Italy. J. Plant Pathol. 2020, 102, 275. [CrossRef] 
47. Alves, A.; Linaldeddu, B.T.; Deidda, A.; Scanu, B.; Phillips, A.J.L. The complex of Diplodia species associated with Fraxinus and some other woody hosts in Italy and Portugal. Fungal Divers. 2014, 67, 143-156. [CrossRef]

48. Dissanayake, A.J.; Camporesi, E.; Hyde, K.D.; Yan, J.Y.; Li, X.H. Saprobic Botryosphaeriaceae, including Dothiorella italica sp nov., associated with urban and forest trees in Italy. Mycosphere 2017, 8, 1157-1176. [CrossRef]

49. Jami, F.; Slippers, B.; Wingfield, M.J.; Loots, M.T.; Gryzenhout, M. Temporal and spatial variation of Botryosphaeriaceae associated with Acacia karroo in South Africa. Fungal Ecol. 2015, 15, 51-62. [CrossRef]

50. Crous, P.W.; Verkley, G.J.M.; Groenewald, J.Z.; Samson, R.A. (Eds.) Fungal Biodiversity; CBS Laboratory Manual Series 1; Centraalbureau Voor Schimmelcultures: Utrecht, The Netherlands, 2009; pp. 1-269.

51. White, T.J.; Bruns, T.; Lee, S.; Taylor, J. Amplification and direct sequencing of fungal ribosomal RNA genes for phylogenetics. In PCR Protocols: A Guide to Methods and Applications; Innis, A.M., Gelfard, D.H., Snindky, J.J., White, T.J., Eds.; Academic Press: San Diego, CA, USA, 1990; pp. 315-322.

52. Carbone, I.; Kohn, L.M. A method for designing primer sets for speciation studies in filamentous ascomycetes. Mycologia 1999, 91, 553-556. [CrossRef]

53. O’Donnell, K.; Kistler, H.C.; Cigelnik, E.; Ploetz, R.C. Multiple evolutionary origins of the fungus causing Panama disease of banana: Concordant evidence from nuclear and mitochondrial gene genealogies. Proc. Natl. Acad. Sci. USA 1998, 95, 2044-2049. [CrossRef] [PubMed]

54. Glass, N.L.; Donaldson, G.C. Development of primer sets designed for use with the PCR to amplify conserved genes from filamentous ascomycetes. Appl. Environ. Microbiol. 1995, 61, 1323-1330. [CrossRef]

55. Katoh, K.; Standley, D.M. MAFFT multiple sequence alignment software version 7: Improvements in performance and usability. Mol. Biol. Evol. 2013, 30, 772-780. [CrossRef] [PubMed]

56. Kumar, S.; Stecher, G.; Tamura, K. MEGA7: Molecular Evolutionary Genetics Analysis Version 7.0 for Bigger Datasets. Mol. Biol. Evol. 2016, 33, 1870-1874. [CrossRef] [PubMed]

57. Stamatakis, A. RAxML version 8: A tool for phylogenetic analysis and post-analysis of large phylogenies. Bioinformatics 2014, 30, 1312-1313. [CrossRef]

58. Nylander, J. MrModeltest v. 2 Program Distributed by the Author; Evolutionary Biology Centre, Uppsala University: Uppsala, Sweden, 2004. 